\title{
A comprehensive view of the Virgo stellar stream ${ }^{\star} \star \star$
}

\author{
Sonia Duffau ${ }^{1,2}$, A. Katherina Vivas ${ }^{3}$, Robert Zinn ${ }^{4}$, René A. Méndez $^{5}$, and María T. Ruiz ${ }^{5}$ \\ 1 Zentrum für Astronomie der Universität Heidelberg, Landessternwarte, Köningstuhl 12, 69117 Heidelberg, Germany \\ e-mail: sduffau@lsw.uni-heidelberg.de \\ 2 Zentrum für Astronomie der Universität Heidelberg, Astronomisches Rechen-Institut, Mönchhofstraße 12-14, \\ 69120 Heidelberg, Germany \\ e-mail: sduffau@ari.uni-heidelberg.de \\ 3 Cerro Tololo Interamerican Observatory, Casilla 603, La Serena, Chile \\ e-mail:kvivas@ctio.noao.edu \\ 4 Department of Astronomy, Yale University, PO Box 208101, New Haven CT 06520-8101, USA \\ e-mail: robert.zinn@yale.edu \\ 5 Departmento de Astronomía, Universidad de Chile, Casilla 36-D, Santiago, Chile \\ e-mail: [rmendez;mtruiz]@das.uchile.cl
}

Received 23 May 2012 / Accepted 26 February 2014

\begin{abstract}
To explore the complex halo substructure that has been reported in the direction of the Virgo constellation, radial velocities and metallicities have been measured for 82 RR Lyrae stars (RRLS) that were identified by the QUEST survey. These stars are distributed over 90 square degrees of the sky, and lie from 4 to $23 \mathrm{kpc}$ from the Sun. Using an algorithm for finding groups in phase space and modeling the smooth halo component in the region, we identified the 5 most significant RRLS groups, some of which were previously known or suspected. We have examined the SEKBO and the Catalina catalog of RRLS (with available spectroscopic measurements), as well as the bright QUEST RRLS sample, the catalog of Red Giant stars from the Spaghetti survey, and three recent catalogs of blue horizontal branch (BHB) stars, for stars that may be related to the QUEST RRLS groups. The most significant group of RRLS is the Virgo stellar stream (VSS) identified here as group A, which is composed of at least 10 RRLS and 3 BHB stars. It has a mean distance of $19.6 \mathrm{kpc}$ and a mean radial velocity $V_{\mathrm{gsr}}=128 \mathrm{~km} \mathrm{~s}^{-1}$, as estimated from its RRLS members. With the revised velocities reported here, there is no longer an offset in velocity between the RRLS in the VSS and the prominent peak in the velocities of main-sequence turnoff stars reported by other researchers in the same direction and at a similar distance (known as S297+63-20.5). The location in phase space of two other groups $(\mathrm{F}$ and $\mathrm{H})$ suggests a possible connection with the VSS, which cannot be discarded at this point, although the turnoff colors of the VSS and group $\mathrm{H}$, as identified from other works, suggest they might be composed of different populations. Two more groups, B and D, are found at mean distances of 19.0 and $5.7 \mathrm{kpc}$, and mean radial velocities of $V_{\mathrm{gsr}}=-94$ and $32 \mathrm{~km} \mathrm{~s}^{-1}$. The latter is the more numerous in terms of total members, as well as the more extended in RA. A comparison with the latest model of the disruption of the Sagittarius dwarf, indicates that none of the above groups is related to it. Rather than being the result of a single accretion event, the excess of stars observed in Virgo appears to be composed of several halo substructures along the same line of sight.
\end{abstract}

Key words. stars: variables: RR Lyrae - Galaxy: abundances - Galaxy: halo - Galaxy: kinematics and dynamics - Galaxy: structure

\section{Introduction}

Over the last decade, a number of simulations of galaxy formation that are based on the popular $\Lambda$ CDM hierarchical picture have predicted that the halos of disk galaxies should contain numerous substructures that are the debris from accreted dwarf galaxies (Bullock \& Johnston 2005; Cooper et al. 2010). Over the same period, the evidence that the Milky Way has indeed accreted dwarf galaxies has grown considerably (e.g. Bell et al. 2008; Helmi et al. 2011; Xue et al. 2011). While there is no doubt that accretion has occurred, it is not yet firm that the number and the properties of the observed substructures are consistent with those simulations. To answer this question, it is not only

* Figure 6 is available in electronic form at http: //www . aanda.org

$\star \star$ Full Tables 5, 6, and 9 are only available at the CDS via anonymous ftp to cdsarc.u-strasbg. fr (130.79.128.5) or via http://cdsarc.u-strasbg.fr/viz-bin/qcat?]/A+A/566/A118 necessary to find the substructures but also to characterize them in sufficient detail that the masses of the dwarf galaxies and the times of their accretion can be estimated. This paper reports a more thorough description of halo substructure in the direction of the constellation Virgo.

After the Sagittarius (Sgr) tidal streams (e.g. Majewski et al. 2003), the Virgo region contains the most obvious overdensity of stars in the sky explored so far by large scale surveys. Discovered as an overdensity of RR Lyrae stars (RRLS) in the QUEST survey (Vivas et al. 2001; Vivas 2002; Vivas \& Zinn 2006; Duffau et al. 2006) and of main-sequence turn-off stars (Newberg et al. 2002) in the SDSS, it is one of the most noticeable features in the Field of Streams (Belokurov et al. 2006). Based in main sequence stars from SDSS, Jurić et al. (2008) estimated that the substructure, covers $\sim 1000 \mathrm{sq} \mathrm{deg}$ of the sky. More recently, Bonaca et al. (2012) suggested the feature may span up to $23000 \mathrm{sq} \mathrm{deg.} \mathrm{Jurić} \mathrm{et} \mathrm{al.} \mathrm{(2008)} \mathrm{suggested} \mathrm{that} \mathrm{the}$ Virgo overdensity (VOD) was produced by the merger of a low 
surface brightness galaxy with the Milky Way (see also Carlin et al. 2012). Spectroscopic observations have revealed, however, a complex system of substructures in the kinematic distribution of stars (Duffau et al. 2006; Newberg et al. 2007; Vivas et al. 2008; Starkenburg et al. 2009; Prior et al. 2009; Brink et al. 2010; Casey et al. 2012). Furthermore, it appears that the strengths of these features depend on the tracer used. For example, the strongest peak in the velocity distribution of RRLS and turnoff stars in the region is found near $V_{\mathrm{gsr}}=120 \mathrm{~km} \mathrm{~s}^{-1}$ (Duffau et al. 2006; Newberg et al. 2007; Prior et al. 2009), while main sequence and $\mathrm{K}$ giants show a peak at $V_{\mathrm{gsr}}=-80 \mathrm{~km} \mathrm{~s}^{-1}$ (Brink et al. 2010; Casey et al. 2012).

In their study of substructures in high resolution simulations of galactic halos from the Aquarius project, Helmi et al. (2011) found that debris from massive progenitors often look like diffuse substructures on the sky. They point out that because of the large-scale structure present in the Universe when galaxies began to form, the accretion of galaxies was not random in direction, but was instead along preferred directions. This infall pattern causes the streams and substructures of different progenitors to overlap. This scenario, rather than a single massive progenitor, may be the explanation for the substructures in Virgo. To complicate the picture, the region of the VOD is not far from the leading tail of the Sgr dSph galaxy. Although the bulk of the Sgr stars lie much farther away, at $50 \mathrm{kpc}$, it has been suggested that stars which became unbound in previous passages of the galaxy may lie at much closer distances (Martínez-Delgado et al. 2007; Prior et al. 2009; Law \& Majewski 2010).

Although the distance to the VOD is relatively small, the spatial distribution of stars alone does not provide a clear picture of the complex substructure. The densest part of the RRLS overdensity lies at $19 \mathrm{kpc}$ (Vivas \& Zinn 2006), but Jurić et al. (2008) suggested that the VOD covered a range of distances from $\sim 5$ to $20 \mathrm{kpc}$. Velocities are key to separate the different accretion events and establish the relationship between them, if any.

In this investigation, we obtained spectroscopy of RRLS present in the QUEST survey in the Virgo region between 4 and $23 \mathrm{kpc}$, with the goal of filling the gap between the earlier investigations by Duffau et al. (2006) and Vivas et al. (2008) who observed QUEST RRLS in ranges $18-20 \mathrm{kpc}$ and $<12 \mathrm{kpc}$, respectively. The sample of RRLS presented here, which is a combination of new observations and an updating of previous ones, includes $87 \%$ of the QUEST RRLS in an area of almost 90 sq degrees of the sky. The relative errors of the RRLS distances are about 7\% (Vivas \& Zinn 2006), which is superior to most other halo tracers (e.g., $\sim 15 \%$ for red giants, Starkenburg 2009) and comparable to that obtained for blue horizontal branch (BHB) stars, their nearest rival. The pulsations of RRLS make the determination of their systemic radial velocities more difficult than for other stars, but even using small numbers of observations of modest precision, it is possible to obtain precisions of $\sim 15-20 \mathrm{~km} \mathrm{~s}^{-1}$ (e.g. Layden 1994; Vivas et al. 2005; Prior et al. 2009). Since the line-of-sight (los) velocity dispersion of the halo is $\sim 115 \mathrm{~km} \mathrm{~s}^{-1}$ over the distance range considered here (Brown et al. 2010), this precision is adequate for identifying the velocity peaks produced by substructure against the background of random halo stars. The main reason to use RRLS as tracers is that even when they do not represent the most numerous population of a system, they provide precise distances and good velocities to help pin-point the location in the sky where to continue searching for evidence of an accretion event. The few RRLS and BHB stars one might find clustered in a particular place in the sky are thus highly significant. The confusion caused by the use of a more numerous tracer with a larger distance error in regions where several stream candidates are suspected can be clarified by the use of RRLS. The trade off is that the number of stars found will be smaller but their parameters will be very reliable.

This paper is organized as follows: in Sect. 2 we describe the sample of RRLS observed here and its relationship (in space) with other works carried out in the region. Section 3 explains the observations and processing techniques that we have used. We look for coherent groups in the sample of RRLS in Sect. 4, and investigate whether or not these groups are also found in surveys using different tracers. Finally, we discuss our results in Sect. 5.

\section{The sample of RR Lyrae stars}

We have merged 3 different spectroscopic data sets: 18 RRLS from Duffau et al. (2006), 29 RRLS from Vivas et al. (2008), and 36 RRLS which are reported here for the first time. In this latest data set most stars come from the QUEST survey (Vivas et al. 2004) but we also took spectra of 3 of the RRLS discovered by Ivezić et al. (2000) (and followed up by Wu et al. 2005), with SDSS data just beyond the northern limit of QUEST (listed in all relevant tables using the prefix W). As we explain below, the radial velocity measurements of the sample RRLS that was observed by Duffau et al. (2006) have been revised. We have also updated the radial velocity measurements for some stars in the sample of Vivas et al. (2008), for which additional photometric and spectroscopic data have been obtained. For three of the stars in our sample we were able to obtain only their mean metallicities, not their radial velocities.

Most of our targets come from the photometric study performed by Vivas and collaborators on two bands of QUEST data. Each band is a $2.2^{\circ}$-wide stripe at a fixed declination observed multiple times in driftscan mode with the QUEST camera (Baltay et al. 2002), with the $1 \mathrm{~m}$ Schmidt telescope at the Venezuelan National Observatory of Llano del Hato. The results of the survey in the band centered at $\mathrm{Dec}=-1^{\circ}$ are reported in Vivas et al. (2004); the second band, at Dec $=-3^{\circ}$, has not been published yet. The selected QUEST RRLS lie at $178^{\circ}<\mathrm{RA}$ $<200^{\circ}$ and $-4.25^{\circ}<$ Dec $<0^{\circ}$, while the 3 SDSS RRLS have the same range of RA but $0^{\circ}<\operatorname{Dec}<1.23^{\circ}$. The RRLS have mean $V$ magnitudes between 13.5 and 17.5 mag, which correspond to a distance from the Sun between 4 and $23 \mathrm{kpc}$. Figure 1 is a polar plot in which right ascension (RA) and distance from the Sun (d) for our sample are shown in the angular and radial direction, respectively.

To compare our sample with previous ones, we show in Figs. 2 and 3 the photometric and spectroscopic detections of substructures in the region. In both figures, the center of the densest part of the overdensity, as seen by the QUEST survey, at $(\mathrm{RA}, \mathrm{Dec})=\left(187^{\circ},-1^{\circ}\right)$, is shown with a $\times$ symbol. Among the detections of substructures in photometric data, there are overdensities of RRLS from the QUEST (Vivas \& Zinn 2006, hereafter V06) and SEKBO surveys (groups K-A and K-B in Fig. 2, Keller et al. 2008), F turnoff stars (Newberg et al. 2002, hereafter N02), main sequence stars in SDSS (Jurić et al. 2008, hereafter J08), and sub-giant stars in SDSS (Keller 2010, hereafter K10). In addition, overdensities have appeared in the region by analyzing the luminosity function (Duffau et al. 2006) and by combining main sequence and red giant stars from the SEKBO survey (Keller et al. 2009, K09). A strong main sequence located at $\sim 23 \mathrm{kpc}$ was detected by Jerjen et al. (2013) in the three fields shown in Fig. 2. One of those fields is coincident with the location of an alleged faint satellite galaxy, Vir Z, discovered by Walsh et al. (2009). However, the results presented by Jerjen et al. (2013) suggest that this object is more extended and 


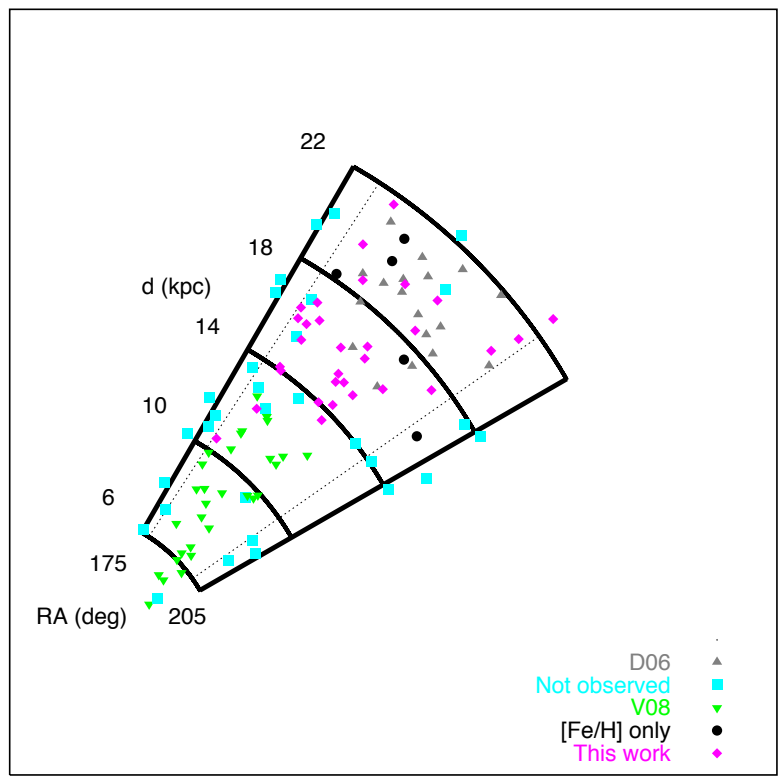

Fig. 1. Distribution of the QUEST RRLS in the sky. The radial direction shows distance from the Sun (d), while the angular direction corresponds to right ascension (RA). Different symbols show the 3 data sets we are using in this work. It can be seen that the new observations (diamond symbols) fill the gap in distance between the samples of Duffau et al. (2006, D06) and Vivas et al. (2008, V08). The square and circle symbols indicate those RRLS for which we did not obtain spectroscopic observations or we were not able to measure a radial velocity. We concentrate our analysis in the region between the two dotted radial lines $\left(178^{\circ}<\mathrm{RA}<200^{\circ}\right)$, in which we have phase space information for most of the QUEST RRLS.

it is located much closer than initially thought. Except for the SEKBO survey, which goes along the ecliptic, all other detections come from QUEST and SDSS, which have a southern limit at about Dec $=-4^{\circ}$ in this part of the sky (shown as a horizontal dotted line in Figs. 2 and 3). Three SDSS/SEGUE stripes cross the region toward southern declinations (also shown as dotted lines). From these stripes and the SEKBO survey, it has been suggested that the VOD extends to the south, beyond the SDSS and QUEST limits (Newberg et al. 2007; Keller et al. 2008; Prior et al. 2009).

Detections of substructure by radial velocity have been made through the measurement of either many stars in relatively small fields, (Newberg et al. 2007; Brink et al. 2010; Casey et al. 2012), or relatively few stars but widely distributed over the region (Duffau et al. 2006; Vivas et al. 2008; Prior et al. 2009; Starkenburg et al. 2009). The latter usually involves targets for which good distance determination is possible. Hence, precise positioning of any stream in the sky is guaranteed, at the expense of a low number of members. Following this idea, Duffau et al. (2006) measured RRLS in the densest part of the overdensity (as seen by QUEST) at $19 \mathrm{kpc}$, and found a coherent velocity at $V_{\mathrm{gsr}} \approx 100 \mathrm{~km} \mathrm{~s}^{-1}$ (but see revision below), which was dubbed the Virgo stellar stream (VSS). Also, the SEKBO survey of RRLS (Prior et al. 2009, hereafter P09), and red giants from the Spaghetti survey (Starkenburg et al. 2009, hereafter S09) found several groups of stars with similar distance and radial velocity. Those groups are shown as circles and triangles connected by lines in Fig. 3 .

Casetti-Dinescu et al. (2009) measured the proper motion of one of the QUEST RRLS within the region of the overdensity, which has a radial velocity consistent with being a member of the

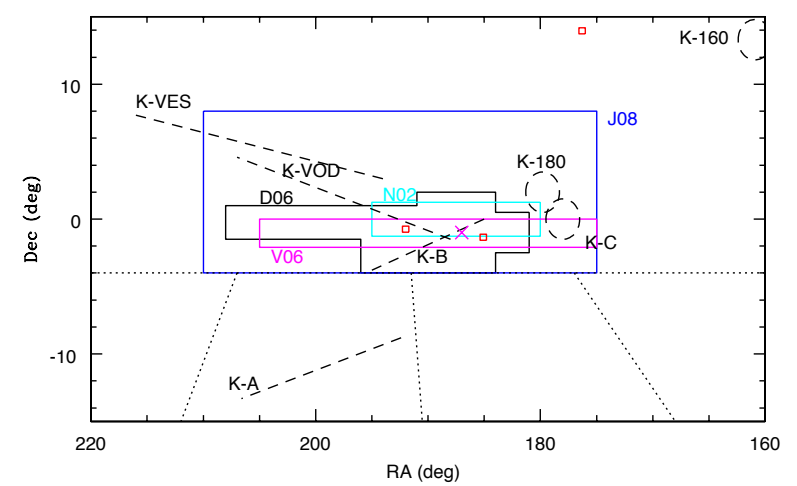

Fig. 2. Distribution in the sky of the different photometric detections of sub-structures in the Virgo region. The dotted horizontal line at $\mathrm{Dec}=-4^{\circ}$ represents the rough southern limit of the SDSS imaging survey. There are also 3 SEGUE imaging stripes that cross the region at negative declinations as shown by the other dotted lines. Labels correspond to the following references: N02, Newberg et al. (2002); V06, Vivas \& Zinn (2006); D06, Duffau et al. (2006); J08, Jurić et al. (2008); K-A, K-B and K-C, Keller et al. (2008, 2009); K-VOD, K-VES, K160 and K-180, Keller (2010). The small squares represent the fields observed by Jerjen et al. (2013); the one at $(\mathrm{RA}, \mathrm{Dec})=\left(185^{\circ},-1.4\right)$ coincides with the location of Vir Z (Walsh et al. 2009). The $\times$ symbol shows the densest part of the overdensity as seen in RRLS in the QUEST survey.

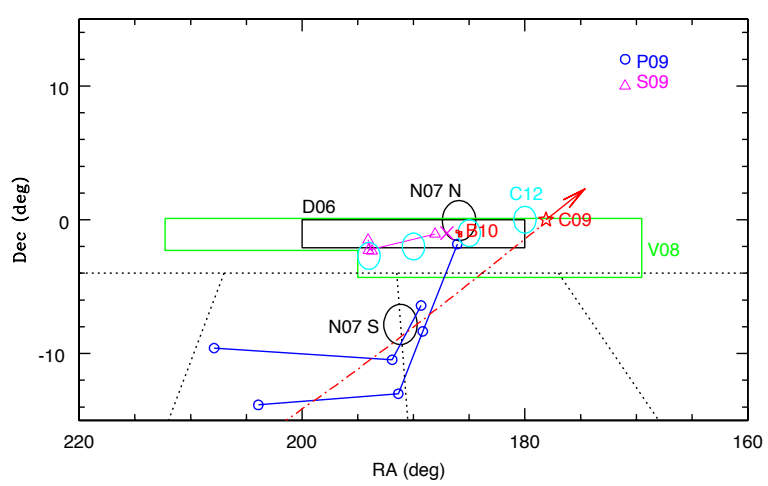

Fig. 3. Similar to Fig. 2 but for kinematic detections. The dotted lines and the $\times$ symbol are the same as in Fig. 2. Labels correspond to the following references: D06 (internal rectangle), Duffau et al. (2006), N07 N and N07 S (large circles), Newberg et al. (2007); V08 (polygon), Vivas et al. (2008); S09 (small triangles), Starkenburg et al. (2009); P09 (small circles), Prior et al. (2009); B10 (tiny squares), Brink et al. (2010); C12 (medium size circles), Casey et al. (2012). The star symbol, arrow and dot-dashed line correspond to the location of an RRLS whose proper motion was determined by Casetti-Dinescu et al. (2009, C09), the direction of the proper motion and part of the past orbit, respectively.

VSS. The measured star and the direction of the proper motion is shown with a star symbol and an arrow in Fig. 3. The path of a tentative past orbit calculated by the authors is shown with a dotdashed lined. This orbit has been confirmed recently by Carlin et al. (2012) using proper motions for a more extended sample of turnoff stars.

\section{Observations}

\subsection{Telescopes and setups}

A total of 179 spectra of 82 RRLS (covering about 90 square degrees in the sky), have been analyzed, including revisions of 
Table 1. Telescopes and Instruments.

\begin{tabular}{lccccccc}
\hline \hline Observatory & Telescope & Instrument & Code & $\begin{array}{c}\text { Grating } \\
\left(\mathrm{mm}^{-1}\right)\end{array}$ & $\begin{array}{c}\text { Spectral range } \\
(\AA)\end{array}$ & $\begin{array}{c}\text { Resolution } \\
(\AA)\end{array}$ & Dates \\
\hline La Silla & $1.5 \mathrm{~m}$ & B\&C & ESO1.5m & 600 & $3300-5500$ & 3.1 & June 2001 \\
La Silla & $3.6 \mathrm{~m}$ & EFOSC2 & ESO3.6m & 600 & $3270-5240$ & 5.4 & Jan.-Feb. 2006 \\
LCO & Clay & B\&C & Mag05 & 600 & $3110-6130$ & 4.3 & March 2005 \\
LCO & Clay & LDSS3 & Mag07 & 1090 & $3440-6140$ & 2.1 & February 2007 \\
CTIO & $4 \mathrm{~m}$ & RC-BAS & $4 \mathrm{~m}$ & 316 & $3480-6460$ & 5.7 & Jan., Mar. 2006 \\
CTIO & $1.5 \mathrm{~m}$ & R-C & SMARTS & 600 & $3532-5300$ & 4.3 & 2003-2004, 2006-2008 \\
KPNO & WIYN & Hydra & WIYN-B & 400 & $3500-6200$ & 7.1 & Apr. 2006, Feb. 2007, Feb. 2008 \\
KPNO & WIYN & Hydra & WIYN-R & 600 & $7100-10000$ & 2.9 & Mar. 2003, Apr. 2007, May 2007 \\
KPNO & WIYN & Hydra & WIYN-G & 600 & $4000-6800$ & 4.6 & May 2008 \\
\hline
\end{tabular}
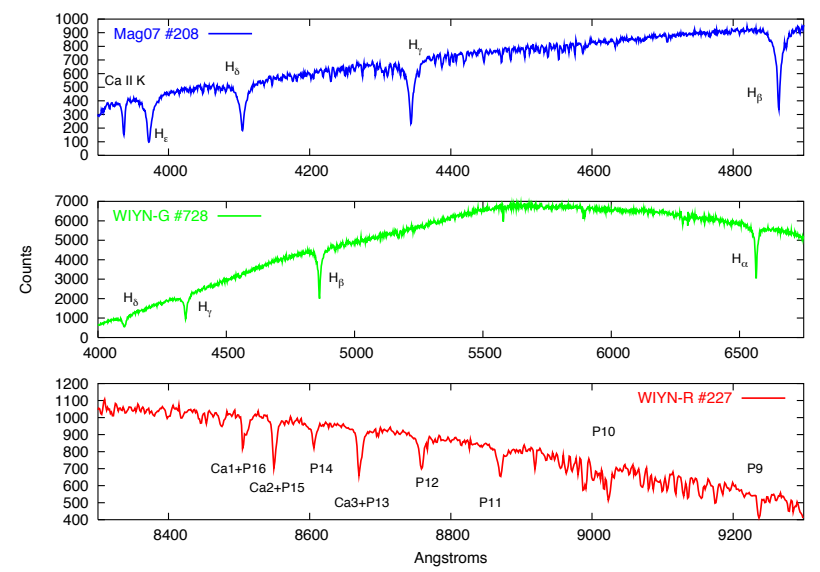

Fig. 4. Example of reduced spectra of RRLS taken with some of the telescopes/setup combinations used for this project. The panels display the three wavelength ranges in use and identify typical spectral features in each of them. The telescope/setup labelling in each panel follows the code defined in Table 1.

previous measurements. The fainter stars were observed with the Magellan, WIYN, Blanco, and ESO 3.6 m telescopes, while we used the $1.5 \mathrm{~m}$ telescopes at both La Silla and CTIO (SMARTS) for the brighter ones. Table 1 displays the details of all the telescopes and instrument setups used in this work. Most of the SMARTS observations were obtained in service mode.

Most of the spectra were taken in the blue part of the optical window, allowing the measurement of Balmer lines (excluding $\mathrm{H} \alpha$ ) and the $\mathrm{Ca}$ II $\mathrm{H}$ and $\mathrm{K}$ lines. However, with the WIYN telescope we used also a "red" and a "green" configuration; this was done mainly to fit some of our targets within other scientific programs at this telescope. In Fig. 4 we show the spectra of stars \#208, \#728 and \#227 which represent spectra in the three different wavelength ranges covered by our telescope/setup combinations. Some characteristic spectral lines of RRLS are marked for reference. Only the blue spectra were used for measuring metallicities, which require a measurement of the $\mathrm{CaII} \mathrm{K}$ line. Typical spectra for this project had a signal to noise of 20 or larger, which is at the same time the minimum we used for estimating metallicities. Radial velocities could be estimated, if necessary, from spectra with a slightly lower signal to noise, but this happened in only a few cases since the quality of the majority of the spectra was high.

\subsection{Observing and reduction techniques}

Since RRLS are pulsating stars, the radial velocity changes during the pulsation cycle. In order to determine the systemic velocity of the star, a curve must be fitted to the radial velocity measurements. We tried to obtain at least two spectra per star, taken at different phases during the cycle, but this was not always possible. As many as 4 spectra were obtained for a few stars.

Since the radial velocity curve of a type ab RRLS has a large discontinuity during the rising branch phase, we avoided taking spectra at phases $>0.85$ and $<0.1$. Because most observations were made in visitor mode, we were able to control the exact phase at which each star was observed using the ephemerides from the QUEST survey. Not only did we avoid the rising branch but we also spaced in phase the observations for the same star for a better fitting of the radial velocity curve. In service mode, all the spectra were taken at random phases. Some of them were not usable because they were obtained on the rising branch, but since this is a rapid phase, only few observations were lost. We did not control the phase of observation in the WIYN/Hydra observations either, because several RRLS in a Hydra field were observed at the same time.

When possible, we took comparison lamp exposures before and after each program star, to account for any flexure of the instrument. In few cases, spectra were reduced using only one of the lamps; in all others, the average of the wavelength calibrations obtained with both lamps was used to calibrate our spectra. The data were reduced using standard IRAF ${ }^{1}$ tasks or packages specifically made for an instrument. Wavelength calibration was performed manually and carefully for each spectrum. The wavelength calibrations had a typical rms of $\sim 2 \mathrm{~km} \mathrm{~s}^{-1}$.

\subsection{New photometry, new ephemerides}

Following the methodology of Vivas et al. (2008), we improved the ephemerides of the QUEST RRLS by adding new photometric data for many of our stars and by fitting an RRLS template to the light curves. The new photometric observations came mainly from QUEST scans taken after the publication of the first catalog. A few additional observations were obtained with the 0.9 SMARTS telescope at CTIO. The fitting of the light curve used $\chi^{2}$ minimization with either templates produced by Layden (1998) for type ab stars, or our own template for type c ones (Vivas et al. 2008). We used the original light curve parameters (namely, period, amplitude, phase at maximum light and maximum magnitude) as initial values and allowed them to vary. The new observations of a few of the type $c$ variables showed that their published periods were spurious. The new light curve

1 IRAF is distributed by the National Optical Astronomy Observatory, which is operated by the Association of Universities for Research in Astronomy (AURA) under cooperative agreement with the National Science Foundation. 
Table 2. Selected Layden stars for use as radial velocity and metallicity standards.

\begin{tabular}{lrr}
\hline \hline Star & $\begin{array}{c}\text { Radial velocity } \\
\left(\mathrm{km} \mathrm{s}^{-1}\right)\end{array}$ & $V$ \\
\hline Feige 56 & 34.6 & 11.1 \\
Kopff 27 & 5.5 & 10.2 \\
HD 693 & 17.0 & 4.9 \\
HD 22413 & 34.8 & 8.7 \\
HD 65925 & -8.2 & 5.2 \\
HD 74000 & 205.6 & 9.4 \\
HD 74438 & 20.4 & 7.9 \\
HD 76483 & 5.4 & 4.9 \\
HD 78791 & 22.4 & 4.5 \\
HD 140283 & -171.4 & 7.3 \\
HD 154417 & -18.0 & 5.8 \\
\hline
\end{tabular}

parameters are part of the table with the final results of this paper (Sect. 4). The new photometry for these stars will be included in an upcoming paper.

For most stars the changes in the light curve parameters were minimal. But even a small change in the date at maximum light may have an effect on the calculation of the phase of a spectrum. There were a few stars (\#174, 180, 201, 203 and 217) for which the changes were more significant. These stars are included in the new La Silla-QUEST survey (Zinn et al. 2014). Although these low amplitude variables were originally classified as type c RRLS in the QUEST catalog Vivas et al. (2004), the light curves of La Silla-QUEST, which have a much larger number of epochs ( $~ 80$ to 190), have long periods and clear signs of asymmetry. The shorter periods assigned by QUEST are aliases of the true ones. These stars were then re-classified as type $b$ and we used the La Silla-QUEST light curve parameters in further calculations.

With these new improved ephemerides we re-calculated the phases at which our old spectra from Duffau et al. (2006) and Vivas et al. (2008) were observed. Consequently, the final radial velocities for these data sets have changed and are updated here.

The La Silla-QUEST data also helped to identify star \#241 (reported as a type $\mathrm{c}$ in Vivas et al. 2004, 2008) as a WUMa eclipsing binary. If the light curve is phased with twice the period reported in our previous work, it is easy to recognize that the two minima have different depths, a clear sign of eclipsing systems. We therefore eliminated \#241 from further analysis.

\subsection{Radial velocities and metallicities}

Except for the case of the red setup for WIYN data, the radial velocities of the target stars were measured with the IRAF task FXCOR. FXCOR performs fourier cross-correlation between a program star and a radial velocity standard star. In the case of the redder WIYN data, we calculated the velocities in a different way, by measuring the centers of some spectral lines. In both cases we followed very closely the methods explained in Vivas et al. (2008), which will not be repeated here. We discuss only relevant changes in the strategy applied specially to the data of the largest telescopes (Magellan and the $3.6 \mathrm{~m}$ telescope).

The cross-correlation technique requires selecting radial velocity standards of similar spectral types as the target to be observed during the corresponding run. For all the runs, we observed several of the radial velocity standard stars from Layden (1994) (from now on simply Layden standards). They are listed in Table 2. Velocities in this table are updated values taken from the SIMBAD database. Not all the standards in Table 2 were observed in every run, but we observed several each night. Some standards were observed several times during the same night or in different nights within the same observing run. The Layden standards have the advantage of serving as metallicity standards as well. We used these standards for cross-correlating all SMARTS and ESO $1.5 \mathrm{~m}$ observations. However, they are bright stars which are hard to observe at large telescopes. During the progress of this work we found the need to set up different standard stars. One set came out of a group of Blue Metal Poor stars studied by Preston \& Sneden (2000) (from now on, Preston-Sneden standards). These standards were observed only in the Mag07 run. The Preston-Sneden standards are closer in magnitude to our target stars. We selected four non variable stars from Preston \& Sneden (2000) which were available in the sky at the time of the observing run. From Preston \& Sneden (2000), these stars have small dispersions in their radial velocities, from $0.52 \mathrm{~km} \mathrm{~s}^{-1}$ to $0.73 \mathrm{~km} \mathrm{~s}^{-1}$.

Finally, we created another set of standards by degrading high resolution spectra obtained from the ELODIE archive (Moultaka et al. 2004) to the resolution of our spectra. We did this for each of the telescope-instrument setups. We refer to this set as the ELODIE standards. These stars have velocity estimates ${ }^{2}$ better than $0.1 \mathrm{~km} \mathrm{~s}^{-1}$. Tables 3 and 4 contain all of the relevant information of the radial velocity standard stars used in these two sets.

For the ELODIE standard set we selected 13 stars with spectral types F, which are similar to the ones of RRLS. To degrade the ELODIE spectra to the proper resolution we used the IRAF task Gauss. These degraded spectra were then binned to match the binning of the targets using dispcor. Finally, we normalized the spectra by using continuum, and trimmed the large spectral range of the ELODIE spectra to match that of the target spectra.

Errors were estimated for each set of standard stars and instrumental setup by cross correlating every standard with all others, and getting the difference between the velocity obtained for each star and its velocity from the literature. This procedure revealed that some of our observations of Layden standard stars were off. Centering these bright stars incorrectly on the slit may have produced a significant shift in the spectrum.

For the Mag07 run we used the Preston-Sneden standards for cross-correlation, and we used the ELODIE standards for the $3.6 \mathrm{~m}$ telescope observations. We then decided to revise the measurements of the stars from the Mag05 run, which were reported in Duffau et al. (2006). The use of ELODIE standards on these observations produced significantly better results, and the new measurements agree with the results obtained by Newberg et al. (2007) for F turnoff stars in the region (see Sect. 4.1).

The estimated error for the radial velocities $\left(\sigma_{\mathrm{r}}\right)$ of the Mag07 run is $13 \mathrm{~km} \mathrm{~s}^{-1}$, while for the $3.6 \mathrm{~m}$ telescope is $9 \mathrm{~km} \mathrm{~s}^{-1}$. The estimated errors from the SMARTS, WIYN (blue, green and red setups) and ESO $1.5 \mathrm{~m}$ telescope data sets are, 16, 8, 6, 9 and $12 \mathrm{~km} \mathrm{~s}^{-1}$, respectively.

Unfortunately, the data from the $4 \mathrm{~m}$ Blanco Telescope at CTIO proved to be unsuitable for radial velocity measurements, as shifts of one pixel or more were found in the raw data on consecutive exposures of the same star. Nevertheless, we could measure the metal abundances of these stars because the typical shifts (of about 1 pixel), represent $\sim 1 \AA$, which is very small compared the width of the spectral features used in Layden's method of determining $[\mathrm{Fe} / \mathrm{H}]$. Using this method, which is only applicable to the blue spectra, we measured $[\mathrm{Fe} / \mathrm{H}]$ with

\footnotetext{
2 Private communication, Sergio Ilovaisky (Observatoire de Haute-Provence).
} 
Table 3. Selected Preston-Sneden stars for use as radial velocity standards.

\begin{tabular}{lcccccc}
\hline \hline Star & $\begin{array}{c}\text { RA } \\
(\mathrm{J} 2000)\end{array}$ & $\begin{array}{c}\text { Dec } \\
(\mathrm{J} 2000)\end{array}$ & $V$ & $(B-V)$ & {$[\mathrm{Fe} / \mathrm{H}]$} & $\begin{array}{c}\text { Radial velocity } \\
\left(\mathrm{km} \mathrm{s}^{-1}\right)\end{array}$ \\
\hline $22175-034$ & 022021.4 & -103810 & 12.6 & 0.34 & -0.28 & 27.2 \\
$22185-009$ & 031453.8 & -144346 & 13.8 & 0.30 & -1.67 & -100.6 \\
$22874-009$ & 143423.0 & -261739 & 13.7 & 0.24 & -0.42 & -36.7 \\
$22874-042$ & 143801.7 & -245847 & 14.0 & 0.33 & -1.53 & 176.2 \\
\hline
\end{tabular}

Table 4. Selected ELODIE stars for use as radial velocity standards.

\begin{tabular}{lcccccr}
\hline \hline Star & $\begin{array}{c}\text { RA } \\
(\mathrm{J} 2000)\end{array}$ & $\begin{array}{c}\text { Dec } \\
(\mathrm{J} 2000)\end{array}$ & $B$ & $V$ & Spectral type & $\begin{array}{r}\text { Radial velocity } \\
\left(\mathrm{km} \mathrm{s}^{-1}\right)\end{array}$ \\
\hline HD 102870 & 115041.7185 & +014552.985 & 4.16 & 3.61 & F9V & 4.43 \\
HD 13555 & 021248.0855 & +211239.575 & 5.64 & 5.24 & F5V & 5.55 \\
HD 136202 & 151918.7977 & +014555.468 & 5.60 & 5.10 & F8III-IV & 54.41 \\
HD 140283 & 154303.0966 & -105600.590 & 7.69 & 7.24 & sdF3 & -169.83 \\
HD 16895 & 024411.9863 & +491342.412 & 4.60 & 4.12 & F7V & 24.44 \\
HD 19994 & 031246.4365 & -011145.964 & 5.63 & 5.06 & F8V & 19.21 \\
HD 222368 & 233957.0409 & +053734.650 & 4.64 & 4.13 & F7V & 5.62 \\
HD 22484 & 033652.3832 & +002405.982 & 4.86 & 4.28 & F9IV-V & 28.08 \\
HD 22879 & 034022.0645 & -031301.133 & 7.23 & 6.74 & F9V & 120.19 \\
HD 3268 & 003554.8015 & +131225.442 & 6.93 & 6.41 & F7V & -23.40 \\
HD 49933 & 065049.8319 & -003227.175 & 6.14 & 5.78 & F2V & -12.57 \\
HD 693 & 001115.8573 & -152804.719 & 5.38 & 4.89 & F8Vfe-08H-05 & 15.04 \\
HD 7476 & 011449.1720 & -005825.661 & 6.12 & 5.70 & F5V & 25.49 \\
\hline
\end{tabular}

Table 5. Individual observations of the target RR Lyrae stars.

\begin{tabular}{lccccccc}
\hline \hline Star & $\begin{array}{c}\text { HJD } \\
(+2450000 \mathrm{~d})\end{array}$ & $\begin{array}{c}\text { Exp } \\
(\mathrm{s})\end{array}$ & Telescope & Phase & $\begin{array}{c}V_{r} \\
\left(\mathrm{~km} \mathrm{~s}^{-1}\right)\end{array}$ & $\begin{array}{c}\sigma_{r} \\
\left(\mathrm{~km} \mathrm{~s}^{-1}\right)\end{array}$ & {$[\mathrm{Fe} / \mathrm{H}]$} \\
\hline W19 & 3820.6861 & 1600 & $4 \mathrm{~m}$ & 0.140 & $\ldots$ & $\ldots$ & -1.62 \\
$\mathrm{~W} 19$ & 4128.8219 & 760 & $\operatorname{Mag} 07$ & 0.276 & 74 & 13 & -1.73 \\
$\mathrm{~W} 21$ & 3821.8407 & 1400 & $4 \mathrm{~m}$ & 0.610 & $\ldots$ & $\ldots$ & -2.18 \\
W31 & 3820.8767 & 1800 & $4 \mathrm{~m}$ & 0.040 & $\ldots$ & $\ldots$ & -1.54 \\
W31 & 4128.8028 & 900 & Mag07 & 0.247 & 112 & 13 & -1.59 \\
172 & 3737.8304 & 520 & ESO3.6m & 0.270 & -25 & 11 & -1.75 \\
174 & 3136.6184 & 3600 & SMARTS & 0.433 & -177 & 15 & $\ldots$ \\
174 & 3137.6494 & 3600 & SMARTS & 0.971 & -207 & 15 & -1.85 \\
175 & 3831.6760 & 1200 & WIYN-B & 0.208 & 180 & 15 & -2.19 \\
175 & 4153.8770 & 3600 & WIYN-B & 0.572 & 292 & 8 & -2.36 \\
\hline
\end{tabular}

Notes. This table is available in its entirety at the CDS. A portion is shown here for guidance regarding its form and content. The table displays the ID of the star, the heliocentric julian date for the observation, the exposure time in seconds, the telescope/setup used, the estimates for phase, radial velocity, radial velocity error, and metallicity. The telescope/setup column corresponds to the codes defined in Table 1 .

precisions of 0.15 and 0.2 dex for types ab and c, respectively. See our previous investigations for more details (Vivas et al. 2005, 2008; Duffau et al. 2006).

Although the dependence of the absolute magnitude of RRLS $\left(M_{V}(R R)\right)$ on metallicity is small, we took advantage of the fact that we had spectroscopic measurements for almost all stars in our sample. We calculated the distances to the stars using the relationship in Demarque et al. (2000):

$M_{V}(R R)=0.22[\mathrm{Fe} / \mathrm{H}]+0.90$.

For the few stars for which we did not measure a metallicity, we assumed $M_{V}(R R)=+0.55$, which corresponds to a metallicity of $[\mathrm{Fe} / \mathrm{H}]=-1.6$ in the above equation.

\section{Results}

Table 5 contains all the individual observations of the RRLS investigated here. The table displays the ID of the star, the heliocentric julian date for the observation, the exposure time in seconds, the telescope/setup used, the estimates for phase, radial velocity, radial velocity error, and metallicity. The telescope/setup column corresponds to the codes defined in Table 1. Many stars have multiple entries in Table 5 since they were observed more than once at different epochs. The table includes the revised velocities of the stars first reported in Duffau et al. (2006). The phase at which the spectra in Duffau et al. (2006) and Vivas et al. (2008) were taken has been recalculated in this table using the new ephemerides.

Figure 5 shows examples of the radial velocity curves fitted to three different stars. The complete sample of radial velocity curves for stars having 2 or more observations are available in the online version of this article (Fig. 6). As can be seen in Fig. 5 (and its online companion), there is good consistency among the results from different instrumental setups, which give us confidence that there are no significant systematic differences among the data sets. Within errors, there is also consistency in 


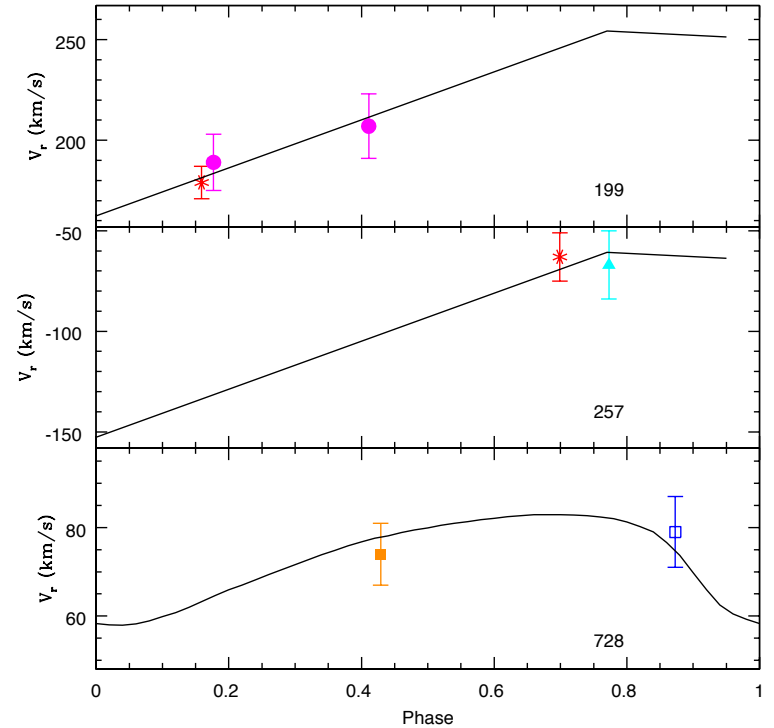

Fig. 5. Example of radial velocity curves fitted to the data of stars 199, 257 and 728. The first two are RRab while the last one (bottom panel) is a type $\mathrm{c}$ star. These stars were observed with different telescopes. The consistency of the data is reassuring that no large systematic offsets are present in the data. Solid circles represent velocities measured in Magellan runs; asterisks, open square and solid square correspond to different WIYN setups (red, blue and green, respectively); triangle is a measurement from the ESO $3.6 \mathrm{~m}$ telescope.

the metallicities determined for the same star from data coming from different telescopes. Table 6 contains the final results for all the 82 stars in the sample, after fitting the radial velocity curves and averaging metallicities obtained from the individual spectra. Because of the revision on velocities and ephemerides, Table 6 supersedes the one in Vivas et al. (2008). Table 6 contains: ID, RA, Dec, mean extinction-corrected magnitude $\left(V_{0}\right)$, period in days, heliocentric Julian day at maximum light $\left(\mathrm{HJD}_{0}\right)$, type of RRLS, distance from the Sun in kpc (d), number of spectra used for the radial velocity curve fitting (Nfit), heliocentric radial velocity $\left(V_{\gamma}\right)$, rms of the fitting of the radial velocity curve $\left(\sigma_{\text {fit }}\right)$, final error in the systemic velocity $\left(\sigma_{\gamma}\right)$, radial velocity in the galactic standard of rest $\left(V_{\mathrm{gsr}}\right)$, and metallicity $[\mathrm{Fe} / \mathrm{H}]$. For details on how the final error in the systemic velocities were calculated we refer the reader to Vivas et al. (2008).

\subsection{Revision of Duffau et al. (2006)}

From the analysis of our first spectroscopic data (18 RRLS and 10 BHB stars from Sirko et al. 2004), we concluded in Duffau et al. (2006) that there was kinematical evidence for a stellar stream (the VSS) at $\sim 19 \mathrm{kpc}$ from the Sun. These first results indicated that a velocity peak at $V_{\mathrm{gsr}} \sim 100 \mathrm{~km} \mathrm{~s}^{-1}$ with a dispersion of $\sigma \sim 17 \mathrm{~km} \mathrm{~s}^{-1}$, and a mean metal abundance estimated in $[\mathrm{Fe} / \mathrm{H}]=-1.86$ and $\sigma=0.40$ existed within the clump of RRLS. The BHB stars in that work were taken from SDSS DR1, as selected by Sirko et al. (2004). We use below the updated velocities of these BHB stars from SDSS DR7.

Figure 7 displays the new velocity histograms for the densest region of the overdensity (at $\sim 19 \mathrm{kpc}$ ), as defined in Duffau et al. (2006). There are 9 stars ( 8 RRLS and 1 BHB star) in this region. In the revised histogram shown in Fig. 7, which can be directly compared with Fig. 2 (top) in Duffau et al. (2006), there

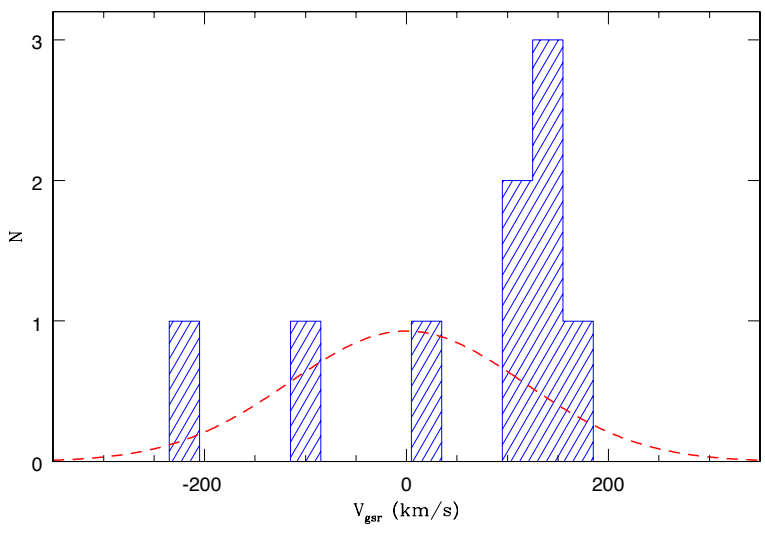

Fig. 7. Radial velocity distribution of 9 RRLS and 1 BHB star in the densest part of the VSS region. For reference, the expected distribution of the same number of halo stars at the distance of this region is shown as a Gaussian curve $\left(\left\langle V_{\mathrm{gsr}}\right\rangle=0 \mathrm{~km} \mathrm{~s}^{-1}, \sigma=116 \mathrm{~km} \mathrm{~s}^{-1}\right)$.

is a clear peak in the velocity distribution, which contains the same 6 stars as in the previous paper, but the peak is slightly shifted toward more positive velocities. The new mean of this kinematical group is $V_{\mathrm{gsr}}=128 \mathrm{~km} \mathrm{~s}^{-1}$ and it has a standard deviation of $\sigma=19 \mathrm{~km} \mathrm{~s}^{-1}$. The mean velocity is now even further away from the model halo without rotation, $\left(0 \mathrm{~km} \mathrm{~s}^{-1}\right)$, and the standard deviation is still only slightly higher than our mean observational error. More importantly, the new mean velocity now agrees very well with the result of $V_{\mathrm{gsr}}=130 \mathrm{~km} \mathrm{~s}^{-1}$ that Newberg et al. (2007) found in the S297+63-20.5 feature from spectroscopy of turnoff F stars. Although S297+63-20.5 and the VSS are virtually in the same part of the sky (see Fig. 2) and at the same distance, Newberg et al. (2007) were reluctant to associate both features because of the offset in velocity. The revision of our velocities, removes the offset to within observational errors. Thus, the VSS and S297+63-20.5 refer to the same halo substructure.

\subsection{Radial velocity distribution in the Virgo region}

Figure 8 shows the distribution of radial velocities for all the stars in the sample. We proceed to examine the velocity histogram for deviations with respect to the expected smooth nonrotating halo model. The mean radial velocity is $31 \mathrm{~km} \mathrm{~s}^{-1}$ with a standard deviation of $124 \mathrm{~km} \mathrm{~s}^{-1}$. The expected distribution of halo field stars at the mean distance of the sample of these RRLS, $\sim 15 \mathrm{kpc}$, has a standard deviation of $\sim 116 \mathrm{~km} \mathrm{~s}^{-1}$, according to the velocity profile measured by Brown et al. (2010), and it is shown by the dashed Gaussian line in Fig. 8. From this figure it is clear that there are important deviations of our data with respect to the expected normal distribution, which suggest that substructures may be present. Because we have taken the complete sample of stars to make this histogram, the information about the distribution in distance of the velocity deviations is not evident.

A better sense of where the kinematic groups may be located can be seen by splitting the velocity histogram into four distance bins (Fig. 9). We examined each bin separately, and found evidence of velocity groups in two of them.

18-23 kpc bin: the most distant bin (top panel in Fig. 9) shows the distribution of the 23 RRLS with $d>18 \mathrm{kpc}$. The distribution of velocities is definitely non-Gaussian and this is confirmed by a Shapiro-Wilk test which rejects normality at 
Table 6. RR Lyrae data: positions, light curve parameters, systemic velocities and metallicity.

\begin{tabular}{|c|c|c|c|c|c|c|c|c|c|c|c|c|c|}
\hline ID & $\begin{array}{l}\text { RA } \\
\text { (deg) }\end{array}$ & $\begin{array}{c}\text { Dec } \\
(\mathrm{deg})\end{array}$ & $V_{0}$ & $\begin{array}{l}\text { Period } \\
\text { (d) }\end{array}$ & $\begin{array}{c}\mathrm{HJD}_{0} \\
(+2450000 \mathrm{~d})\end{array}$ & Type & $\begin{array}{c}d \\
(\mathrm{kpc})\end{array}$ & Nfit & $\begin{array}{c}V_{\gamma} \\
\left(\mathrm{km} \mathrm{s}^{-1}\right)\end{array}$ & $\begin{array}{c}\sigma_{\text {fit }} \\
\left(\mathrm{km} \mathrm{s}^{-1}\right)\end{array}$ & $\begin{array}{c}\sigma_{\gamma} \\
\left(\mathrm{km} \mathrm{s}^{-1}\right)\end{array}$ & $\begin{array}{c}V_{\mathrm{gsr}} \\
\left(\mathrm{km} \mathrm{s}^{-1}\right)\end{array}$ & {$[\mathrm{Fe} / \mathrm{H}]$} \\
\hline W19 & 180.199665 & 0.769750 & 17.00 & 0.34347 & 2698.87810 & $\mathrm{c}$ & 19.7 & 1 & 77 & 0 & 15 & -25 & -1.68 \\
\hline W21 & 183.373500 & -1.031083 & 17.15 & 0.61212 & 3053.86830 & $a b$ & 22.2 & $\ldots$ & $\ldots$ & $\ldots$ & $\ldots$ & $\ldots$ & -2.18 \\
\hline W31 & 190.402665 & 1.218472 & 17.04 & 0.60512 & 3062.42510 & $a b$ & 19.9 & 1 & 142 & 0 & 20 & 63 & -1.59 \\
\hline 172 & 180.185025 & -0.712449 & 16.54 & 0.52119 & 1610.71317 & $a b$ & 16.0 & 1 & 2 & 0 & 17 & -104 & -1.75 \\
\hline 174 & 180.397425 & -0.234712 & 16.09 & 0.67010 & 5276.62778 & $a b$ & 12.7 & 1 & -169 & 0 & 20 & -273 & -1.85 \\
\hline 175 & 180.632505 & -1.895691 & 16.14 & 0.57091 & 1582.74254 & $a b$ & 14.1 & 2 & 249 & 34 & 34 & 140 & -2.28 \\
\hline 176 & 181.087335 & -2.178318 & 16.38 & 0.33061 & 1971.84326 & $\mathrm{c}$ & 14.4 & 2 & 385 & 4 & 7 & 276 & -1.41 \\
\hline 177 & 181.212600 & -0.351735 & 17.03 & 0.37668 & 1585.81581 & $\mathrm{c}$ & 21.0 & 2 & 261 & 3 & 13 & 158 & -2.19 \\
\hline 178 & 181.517325 & -2.215837 & 15.10 & 0.69927 & 2002.62849 & $a b$ & 8.5 & 2 & 85 & 3 & 14 & -23 & -2.08 \\
\hline 180 & 181.911345 & -0.752715 & 15.97 & 0.67196 & 5287.51756 & $a b$ & 10.7 & 1 & 193 & 0 & 20 & 90 & -1.26 \\
\hline
\end{tabular}

Notes. This table is available in its entirety at the CDS. A portion is shown here for guidance regarding its form and content. See text for description.

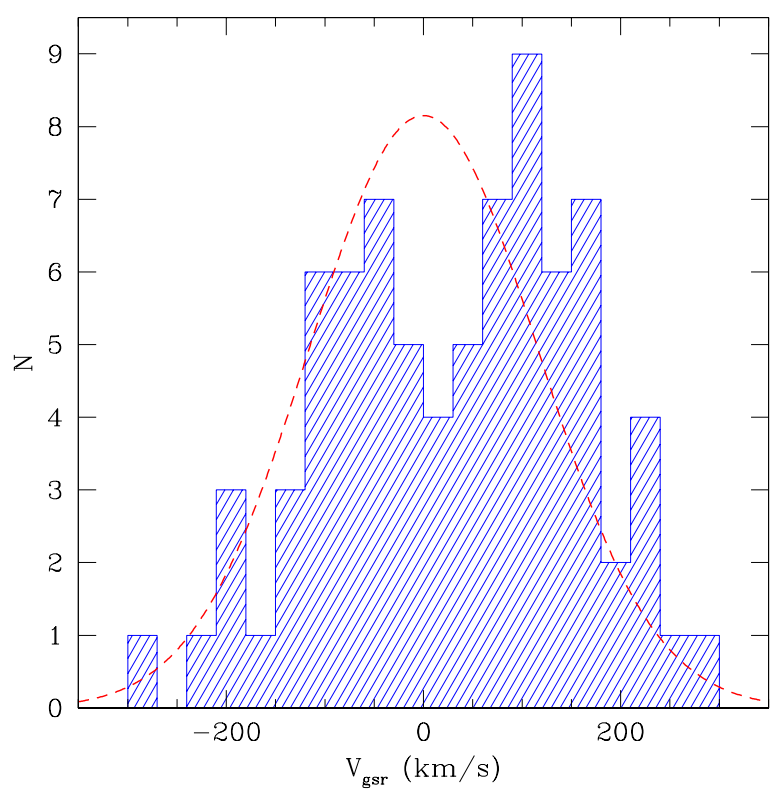

Fig. 8. Radial velocity distribution of 79 RRLS in the Virgo region. Bin size is $30 \mathrm{~km} \mathrm{~s}^{-1}$. For comparison, the expected distribution of the same number of halo stars at the average distance of these RRLS is shown as a Gaussian curve $\left(\left\langle V_{\mathrm{gsr}}\right\rangle=0 \mathrm{~km} \mathrm{~s}^{-1}, \sigma=116 \mathrm{~km} \mathrm{~s}^{-1}\right)$.

the $97 \%$ confidence level. This panel corresponds to the range of distances where the VSS was first detected by Duffau et al. (2006). The VSS is seen in this histogram as 7 stars with $120 \lesssim$ $V_{\mathrm{gsr}} \lesssim 180 \mathrm{~km} \mathrm{~s}^{-1}$. But, with the inclusion of more stars to the original Duffau et al.'s sample, a second peak in the distribution is now easily observed at $V_{\mathrm{gsr}} \sim-75 \mathrm{~km} \mathrm{~s}^{-1}$.

10-14 kpc bin: the distribution of velocities in the range of distances $10<d<14 \mathrm{kpc}$ also shows hints of substructures since an excess of stars at very high positive velocity $\left(>150 \mathrm{~km} \mathrm{~s}^{-1}\right)$ is present. This peak is mostly made out of the stars already reported in Vivas et al. (2008). A second peak is seen at $V_{\mathrm{gsr}}=105 \mathrm{~km} \mathrm{~s}^{-1}$. In this distance bin, normality in the velocity distribution is also rejected by the Shapiro-Wilk test at the $95 \%$ confidence level. The stars in this bin have a mean velocity $\left\langle V_{\mathrm{gsr}}\right\rangle=57 \mathrm{~km} \mathrm{~s}^{-1}$, which is significantly offset from the nominal value of the halo; this is of course due to the presence of the two positive velocity groups. The standard deviation is also significantly larger than the nominal halo, $\sigma=166 \mathrm{~km} \mathrm{~s}^{-1}$.

Bins 4-10 kpc and 14-18 kpc: these two bins do not show significant features in the velocity distribution. According to Shapiro-Wilk statistical test there is no reason to suspect the stars in these bins are not drawn from a normal distribution.

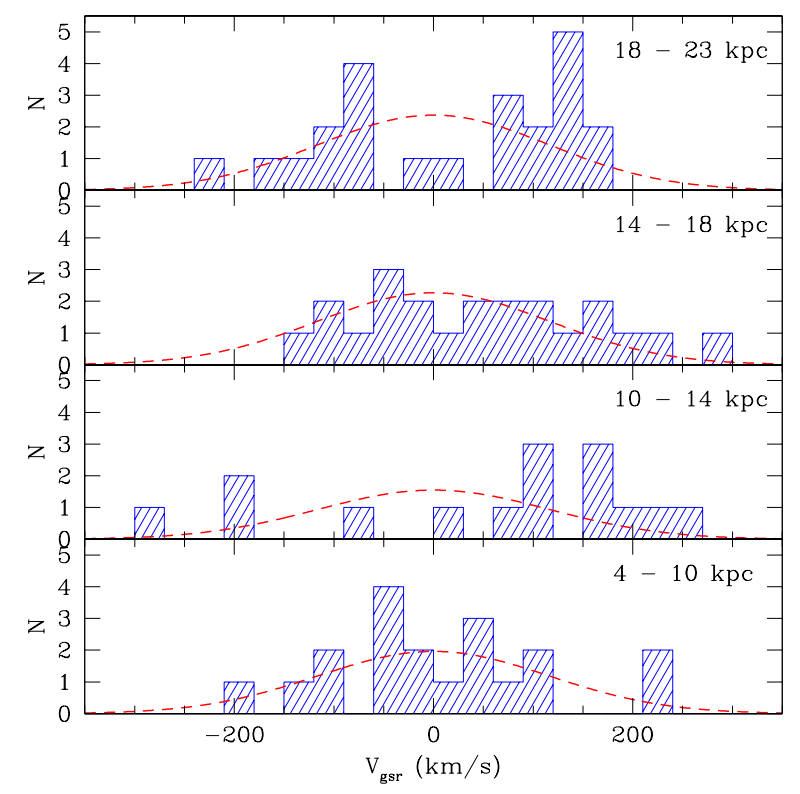

Fig. 9. Radial velocity distribution of our stars in 4 different distance bins. Gaussian curves representing a halo smooth distribution are shown for the same number of stars contained in each distance bin.

Since we chose arbitrarily the limits of the distance bins in the four panels of Fig. 9, it is possible that the identified velocity groups extend beyond the limits where they are recognized. In the following sections, we will apply a group finding algorithm to the RRLS without imposing distance bins.

\subsection{Group finding algorithm}

To find groups of stars in phase space we have used the idea of stellar pairs, inspired by the works of Clewley \& Kinman (2006), Vivas et al. (2008) and Starkenburg et al. (2009). Two stars make a stellar pair when they are close in space and their velocities are similar. A group is formed when two or more pairs have stars in common. Thus, the minimum number of members in a group is 3 (two pairs with 1 star in common).

Starkenburg et al. (2009) define a "pair" of stars based on the four-distance, $4 d_{i j}$, between stars $i$ and $j$ :

$\left(4 d_{i j}\right)^{2}=\omega_{\phi}^{2} \phi_{i j}^{2}+\omega_{d}\left(d_{i}-d_{j}\right)^{2}+\omega_{v}\left(v_{i}-v_{j}\right)^{2}$

where the first term on the right hand of the equation represents the angular distance of two stars in the sky, and the second and third terms are their differences in distance from the Sun and 
radial velocity, respectively. Each term is weighted by the values $\omega_{\phi}, \omega_{d}$ and $\omega_{v}$, defined in Starkenburg et al. (2009), to take into account the observational errors and to normalize to the maximum possible separation between the two stars. With this definition, two stars make a pair when $4 d_{i j}<\epsilon$, where $\epsilon$ is a free parameter that gives a measurement of how tight the groups are in phase space.

A potential problem with the above formalization is that the maximum allowed separation in the sky (measured in degrees) means different physical scales of the projected size in kpc of a group of stars in the sky, depending on their distance from the Sun. This means that with increasing distance, the method imposes the search of groups which are tighter in the direction perpendicular to the line of sight compared with the allowed size along the line of sight. This is not appropriate for the search of cold streams, where the constraint in distance separation must remain the same along the stream independent of orientation or distance from the Sun.

Here we propose a modification on the definition of the fourdistance to ensure that at any place in the Galactic halo, the method does not impose any preference to the physical scale along one dimension or the other:

$\left(4 d_{i j}\right)^{2}=\omega_{3 d_{i j}}^{2}\left(3 d_{i j}\right)^{2}+\omega_{v}\left(v_{i}-v_{j}\right)^{2}$

where $3 d_{i j}$ is the three-dimensional distance between two stars calculated from their galactocentric cartesian coordinates $(x, y, z)$ :

$3 d_{i j}=\sqrt{\left(x_{i}-x_{j}\right)^{2}+\left(y_{i}-y_{j}\right)^{2}+\left(z_{i}-z_{j}\right)^{2}}$.

The weights in Eq. (3), $\omega_{3 d_{i j}}$ and $\omega_{v}$, are defined following Starkenburg et al. (2009) as:

$$
\begin{aligned}
\omega_{3 d_{i j}} & =\left(\frac{1}{(3 d)_{\max }}\right)^{2} \frac{\left(\frac{\sigma d_{i}}{d_{i}}\right)^{2}+\left(\frac{\sigma d_{j}}{d_{j}}\right)^{2}}{2\left\langle\frac{\sigma d}{d}\right\rangle^{2}} \\
\omega_{v} & =\left(\frac{1}{v_{\max }}\right)^{2} \frac{\sigma v_{i}^{2}+\sigma v_{j}^{2}}{2\langle\sigma v\rangle^{2}} .
\end{aligned}
$$

The weights are normalized by the maximum possible range in distance and velocity for two stars which we set to $(3 d)_{\max }=$ $60 \mathrm{kpc}$ and $v_{\max }=550 \mathrm{~km} \mathrm{~s}^{-1}$. With a $\sigma$ of $116 \mathrm{~km} \mathrm{~s}^{-1}$ (Brown et al. 2010) for the distribution of the radial velocities of halo stars, the value of $v_{\max }$ encloses $2.2 \sigma$ of the distribution. On the other hand, the number of RRLS farther away than $60 \mathrm{kpc}$ from the galactic center is very low (Sesar et al. 2007; Zinn et al. 2014) and thus it is an appropriate value for $(3 d)_{\max }$. For the weight in the $(3 \mathrm{~d})$ distance we only took into account the error in distance along the line of sight since the errors in position of the stars (less than $1^{\prime \prime}$ ) are negligible compared with the typical separation between stars in a pair (a few degrees).

The critical value $\epsilon$ establishes how small is the separation of two stars in space and in velocity. It is a measure of the scale of clumpiness of the groups. The smaller the values of $\epsilon$, the tighter the groups of stars. Table 7 shows the maximum values in $3 d$ distance and velocity for stars in a pair for different values of $\epsilon$, which are obtained by making two stars having one of the parameters (either $3 d$ distance or velocity) exactly the same. In practice stars in a pair will have separations smaller than the values quoted in the table since it is unlikely to have two stars with exactly the same position or velocity. Small values of $\epsilon$ may impose constraints which are too tight for the expected density of stars in a stream, velocity dispersion of a typical stream, and for
Table 7. Maximum values for stars in a pair.

\begin{tabular}{lcc}
\hline \hline$\epsilon$ & $3 d(\mathrm{kpc})$ & $v\left(\mathrm{~km} \mathrm{~s}^{-1}\right)$ \\
\hline 0.02 & 1.2 & 10 \\
0.03 & 1.8 & 15 \\
0.04 & 2.4 & 20 \\
0.05 & 3.0 & 25 \\
0.06 & 3.6 & 30 \\
\hline
\end{tabular}

the observational errors of our sample. For example, the maximum separation in velocity of a pair of stars with $\epsilon=0.02$ is only $10 \mathrm{~km} \mathrm{~s}^{-1}$, which is smaller than our observational errors. On the other hand, very large values of $\epsilon$ may be unable to distinguish individual groups, since they will pair the whole sample into one big group.

For our RRLS sample we explored different values of $\epsilon$ in the range from 0.02 to 0.07 and we found pairs and groups with all of them. We show in Fig. 10 some examples of the groups we found with three different values of $\epsilon(0.04,0.046$ and 0.06$)$. The plots show the distribution in phase space of each one of the groups that were detected with a different color/symbol. The same group may be detected with different values of $\epsilon$. A tight group (small $\epsilon$ ) may get more members with increasing $\epsilon$ (for example, the solid green squares at $\sim 130 \mathrm{~km} \mathrm{~s}^{-1}$ ), unless it is physically a very concentrated and isolated clump in phase space (for example, the solid cyan stars at $\sim 40 \mathrm{~km} \mathrm{~s}^{-1}$ ). Other groups just appeared for the first time with the largest value of $\epsilon$. In the figure it can be seen that both the number of groups and the number of members in each group increase with $\epsilon$, until $\epsilon=0.06$ where the number of groups starts to decline. This is a sign that the maximum allowed separations in distance and velocity are too large and several groups have merged together. In addition, for $\epsilon \geq 0.05$ we obtained several groups that have velocity dispersions larger than the expectations for cold substructures in the halo (which should be below $\sim 30 \mathrm{~km} \mathrm{~s}^{-1}$ ). Some groups also have an unusually large range in distance along the line of sight, covering in some cases almost the whole range of the RRLS sample. Examples of this can be seen in the lower panel of Fig. 10 which shows the results for $\epsilon=0.06$. Some of the groups (for example, the red open circles at $\sim-75 \mathrm{~km} \mathrm{~s}^{-1}$ ) are just too dispersed both in distance and velocity to believe they are part of a real cold substructure. Thus, the optimal value of $\epsilon$ for finding cold substructures in the RRLS samples seems to be a value below 0.05 .

We found that $\epsilon=0.046$ allowed for the loosest constraints that produced the maximum number of members in each group but still having velocity and distance dispersions expected for halo substructures. In the following we study with detail the groups found with this value of $\epsilon$. Table 8 indicates the mean properties of the 8 groups (labelled with letters $\mathrm{A}$ to $\mathrm{H}$ ) detected with this value of $\epsilon$, including mean distance and velocity, velocity dispersion, distance dispersion (along the line of sight) and number of members. In addition, we calculated the $3 d$ dispersion, $\sigma 3 d$, of each group by obtaining the coordinates of the center of the group from the average of the cartesian coordinates of the members. Then, we estimated the distance separation of each member to the center of the group. The $3 d$ dispersion, which is given in $\mathrm{kpc}$, is the average of those distance separations. Finally the table contains some statistics that help to determine if the group is a significant feature of the halo, as we explain in the next section. 
A\&A 566, A118 (2014)

Table 8. Properties of the groups of RRLS in the Virgo region detected with $\epsilon=0.046$.

\begin{tabular}{lccccccccccc}
\hline \hline Group & $\begin{array}{c}\text { Color/ } \\
\text { symbol }\end{array}$ & $N$ & $\begin{array}{c}\left\langle V_{\mathrm{gsr}}\right\rangle \\
\left(\mathrm{km} \mathrm{s}^{-1}\right)\end{array}$ & $\begin{array}{c}\sigma V_{\mathrm{gsr}} \\
\left(\mathrm{km} \mathrm{s}^{-1}\right)\end{array}$ & $\begin{array}{c}\langle d\rangle \\
(\mathrm{kpc})\end{array}$ & $\begin{array}{c}\sigma d \\
(\mathrm{kpc})\end{array}$ & $\begin{array}{c}\sigma 3 d \\
(\mathrm{kpc})\end{array}$ & $P\left(V_{\mathrm{gsr}}\right)$ & $P(\mathrm{~d})$ & $P(\sigma d)$ & $P(\sigma 3 d)$ \\
\hline $\mathrm{A}$ & green/solid squares & 9 & 128 & 18.9 & 19.7 & 1.15 & 1.57 & 99.6 & 98.8 & 11.3 & 28.7 \\
$\mathrm{~B}$ & red/open circles & 5 & -89 & 14.7 & 18.8 & 0.29 & 1.42 & 79.2 & 90.9 & 0.6 & 58.1 \\
$\mathrm{C}$ & magenta/solid circles & 5 & -41 & 10.6 & 9.0 & 0.72 & 0.95 & 43.3 & 37.7 & 15.4 & 13.3 \\
$\mathrm{D}$ & cyan/solid stars & 4 & 43 & 11.3 & 5.4 & 0.86 & 0.81 & 39.4 & 3.2 & 40.3 & 12.1 \\
$\mathrm{E}$ & blue/solid triangles & 4 & 95 & 5.5 & 11.2 & 0.75 & 0.80 & 77.0 & 50.4 & 29.8 & 11.6 \\
$\mathrm{~F}$ & black/open stars & 4 & 222 & 12.8 & 10.6 & 1.14 & 1.45 & 99.8 & 46.4 & 66.0 & 75.3 \\
$\mathrm{G}$ & blue/open triangles & 3 & 57 & 7.4 & 16.5 & 0.33 & 1.05 & 46.3 & 72.4 & 11.4 & 52.0 \\
$\mathrm{H}$ & pink/open squares & 3 & 173 & 6.0 & 12.6 & 1.01 & 1.11 & 96.4 & 51.9 & 72.7 & 59.3 \\
\hline
\end{tabular}

Notes. $N$ is the number of members of the indicated group, $P$ (variable) is the percentile for that group in that particular variable. See text for description.

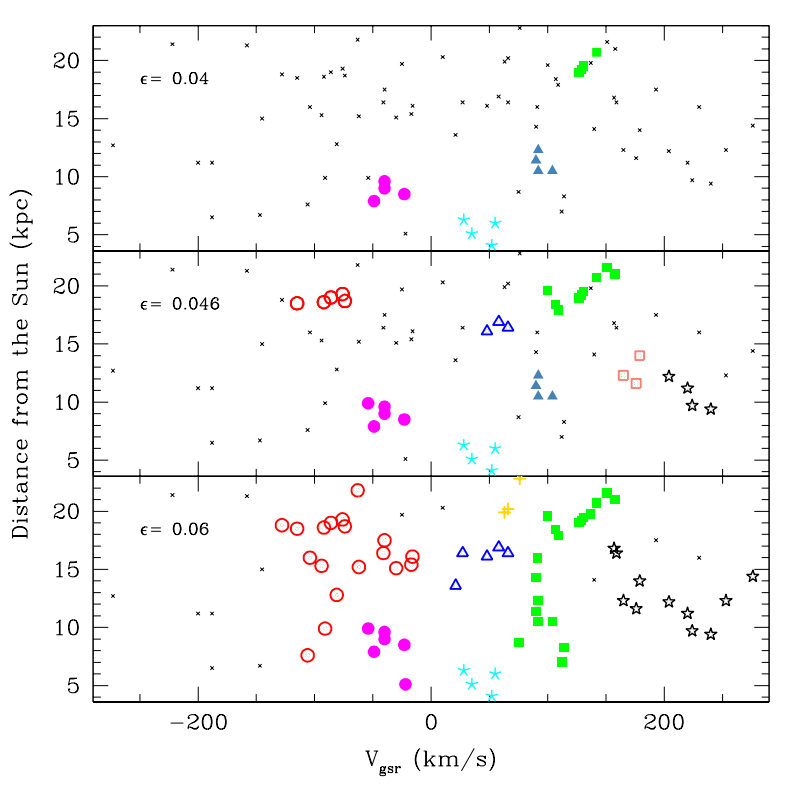

Fig. 10. Distance from the Sun versus radial velocity for all the RRLS in the sample. Groups detected with $\epsilon=0.04$ (top), 0.046 (middle) and 0.06 (bottom) are shown with different colors/symbols. The properties of the groups shown in the middle panel $(\epsilon=0.046)$ are specified in Table 8 .

\subsection{Simulations}

A smooth distribution of stars in the sky could produce groups just by random fluctuations. In order to test if our detected groups are special features in the halo or just random fluctuations in a smooth distribution, we performed extensive simulations of random samples of stars. The simulated samples have the following properties:

- Each sample has a random number of stars taken from a Poisson distribution with mean $N$. The value of $N$ was obtained by integrating the number density profile of RRLS in the halo with the limits in area and depth similar to the observed sample. We obtained that this region of the halo should contain $N_{\mathrm{ab}}=42$ from the halo density profile given in Vivas \& Zinn (2006) for type ab stars. By assuming that $N_{\mathrm{ab}+\mathrm{c}} / N_{\mathrm{c}}=1.29$ (Layden 1995$)$, we obtain a total number of RRLS (all types) of $N=54$. This number is significantly lower than the number in our sample of RRLS (79), which is expected because the Vivas \& Zinn (2006) profile was measured in regions that do not contain obvious halo substructures, whereas several authors have found evidence of substructures (see above) in the region under study. These substructures are expected to be imprinted on a smooth background of unrelated RRLS. We have used $N=54$ in our simulations to see if random fluctuations of the background alone can account for any of the groups identified in Table 8.

- A distance was assigned to each star by randomly sampling the density profile of RRLS given in Vivas \& Zinn (2006) between 4 and $22 \mathrm{kpc}$. A random error of $7 \%$, similar to the error of our observations, was added.

- Random coordinates (RA, Dec) were assigned to each star within the limits of the survey area.

- Radial velocities were drawn from a Gaussian distribution with mean $0 \mathrm{~km} \mathrm{~s}^{-1}$ and $\sigma_{\text {los }}=116 \mathrm{~km} \mathrm{~s}^{-1}$, which is the value derived by Brown et al. (2010) for stars at $\sim 15 \mathrm{kpc}$ from the Sun. A random error of $16 \mathrm{~km} \mathrm{~s}^{-1}$ was added to each simulated star.

This way, we created 10000 simulated samples and we detected pairs and groups in each one of them using exactly the same procedure as with the RRLS sample. Pairs and groups appeared frequently in the simulated samples. There are enough mock groups in our simulations to test statistically their properties and compare them to our observed groups. In the case of $\epsilon=0.046$, for example, there are $\sim 36000$ groups formed in the simulations (an average of $\sim 3.6$ groups per simulation in 10000 simulations). Our goal is to see if any of the RRLS groups have properties that are not easily reproduced by the random groups.

Figure 11 shows some of the properties of the groups found in the simulated samples with $\epsilon=0.046$. Panel (a) shows that most of the mock groups contain only 3 members. The number of mock groups decreases by a factor of $\sim 2$ for $N_{\text {stars }} \geq 4$. In the groups of RRLS we have 2 groups having 3 members each, 3 groups with 4 members, 2 with 5 and 1 with 9 members. It is very rare $(<3 \%)$ in our simulations to form a group with 9 or more members. Group A with 9 members is hence, significant.

Figure $11 \mathrm{~b}$ shows the absolute value of the mean radial velocity of the simulated groups. In this plot the lines indicate the percentiles 75, 90 and 95 in the distribution of velocities of groups with a given $N$. Most of the groups in the simulated samples have small velocities which agrees with the distribution of velocities given to the random stars. Groups with small $N$ may form with relatively large velocities but with increasing $N$ the largest velocity possible in the groups decreases. The solid points in Fig. $11 \mathrm{~b}$ indicate the velocities of the eight groups of RRLS indicated in Table 8. Three of those groups (A, F and $\mathrm{H}$ ) have velocities which are above the percentile 95 of the distribution. Groups $\mathrm{F}$ and $\mathrm{H}$ have only 4 and 3 members respectively. 

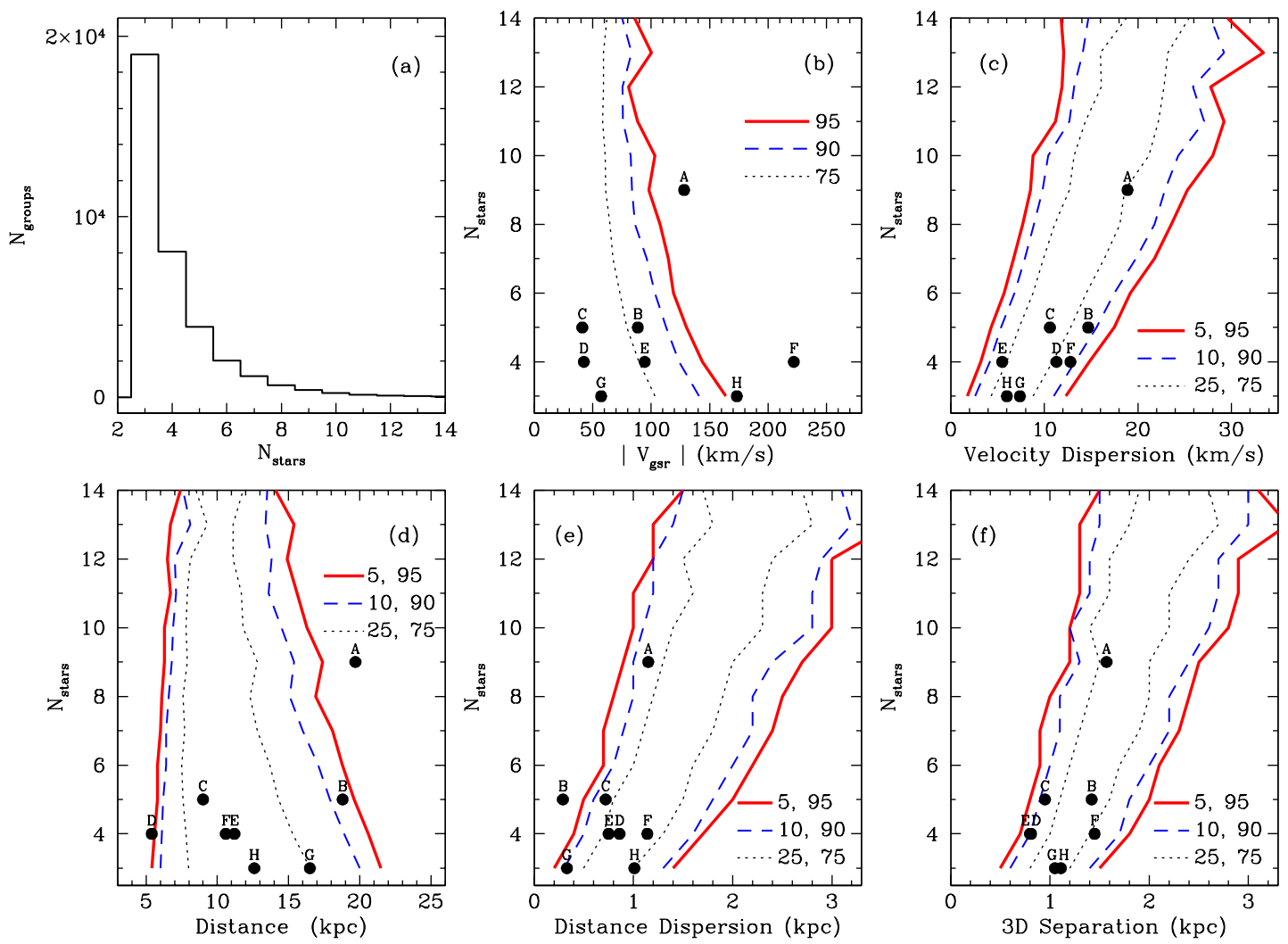

Fig. 11. Properties of the groups found in the simulated samples using $\epsilon=0.046$. a) Distribution of the number of stars in the groups. Lines in panels b)-f) indicate the percentiles 5, 10, 25, 75, 90 and 95 of the distribution of radial velocity b), velocity dispersion c), distance d), distance dispersion e) and 3D separation f), for groups having $N_{\text {stars }}$ members. For the velocity distribution b) the $x$ axis shows the absolute value of the velocity and hence, only the percentiles 75, 90 and 95 are indicated. Panels b) to f) show the location of groups A to $\mathrm{H}$ (Table 8) as solid circles.

Although it is very common to have modelled groups with this low number of stars, it is very rare that they have such a high radial velocity. This fact makes these groups strong candidates to be real features of the halo and not just random fluctuations.

The rest of the panels in Fig. 11 shows the distribution of other properties measured in the random groups, namely, velocity dispersion (c), distance (d), distance dispersion (e) and mean separation (3D) from the center of the group (f). For these properties we also show the lines of the percentiles 5, 10 and 25. The mean distance of the groups (Fig. 11d) seems to be another parameter that allow us to recognize special features over random fluctuations. At every distance is relatively easy to form groups of small $N$, but with increasing distance, the decline in the density of RRLS makes it increasingly difficult to form groups of large $N$. Again, group A, at $19.7 \mathrm{kpc}$ from the Sun, seems special since it is above the 95th percentile of the distance distribution for groups with 9 members. In addition, group B $(5$ members, $18.8 \mathrm{kpc}$ ) is above the 90th percentile for groups of such $N$.

Group B also stands out because the distance dispersion (Fig. 11e) is significantly smaller than any of the random groups. This group has not a particularly small 3D separation, suggesting that the group is more concentrated along the line of sight than across the sky. This may be understood if the stream is crossing almost perpendicularly our line of sight.

\subsection{Significant groups}

The analysis of the properties of the simulated groups indicate that some of our groups of RRLS cannot be explained as random fluctuations in the distribution of halo stars. We considered that a group is potentially significant if at least one of their properties is below or above the percentiles 10 and 90 of the distribution of simulated groups for a given $N$. This way, five of the groups of RRLS have properties that are quite different from groups formed by chance among halo stars. This five groups are thus strong candidates to be real substructures of the halo.

Without a doubt, group $\mathrm{A}$ is the most significant feature found in this part of the sky. First it has a very high number of members, which is unusual in the random groups formed in the simulated samples. Furthermore, from the few groups formed with 9 members, none has the combination of mean velocity and distance as group A. This group corresponds to the VSS as first determined by Duffau et al. (2006) and Newberg et al. (2002). The center of the group is found at $\mathrm{RA}=186.2^{\circ}$, but it contains stars basically throughout the region explored, from $180^{\circ}$ to $195^{\circ}$.

Group B has 5 members and it is also located at a large distance from the Sun, $18.8 \mathrm{kpc}$. This is above the 90th percentile of the distance distribution for groups with $N=5$. Group B has another remarkable property and it is that it has a very small dispersion along the line of sight, of only $0.29 \mathrm{kpc}$, which may be an indication of a thin stream crossing the region perpendicular to the line of sight.

Groups $\mathrm{F}$ and $\mathrm{H}$, with 4 and 3 members respectively, have velocities unusually high which make them significant even when having such low number of members. Group F is the same reported already by Vivas et al. (2008).

Finally, group D is unusual because it is formed by the stars closest to the Sun in our sample. It is remarkable that out of the 5 stars with distances closer than $6.5 \mathrm{kpc}, 4$ of them make a 
tight group. This group is present even with a low value of $\epsilon$ of only 0.035 .

The remaining groups, $\mathrm{C}, \mathrm{E}$ and $\mathrm{G}$ may not be significant and thus are not discussed further.

Figure 11 shows that it would be hard to use this method for detecting groups having very low $\left|V_{\mathrm{gsr}}\right|$ since they are easily formed among field stars. In order to be detected by this method, such groups should have an unusual property such as a very small dispersion in velocity and/or distance.

\subsection{Comparison with other catalogs and tracers}

Several samples of halo stars with both reliable distances and radial velocities are available in the literature. We used those sources to find additional evidence for the kinematical groups described above. We used the new spectroscopic catalog of bright QUEST RRLS, which contains velocity measurements for all the stars in that catalog brighter than $V \sim 16$ (Vivas et al., in prep.). We included in this catalog the velocity measurement of QUEST star \#167 made by Casetti-Dinescu et al. (2009), which was associated with the VSS by those authors. We also compared our results with a subset of RRLS in Virgo from the SEKBO and the Catalina catalogs which have spectroscopic information (Prior et al. 2009; Drake et al. 2013). In the case of Catalina, we found 14 stars in common with the QUEST catalog and a comparison of the velocities indicates a systematic offset of $27 \mathrm{~km} \mathrm{~s}^{-1}$ between both catalogs, with the QUEST velocities being larger than Catalina's. For the purposes of this work, we added $27 \mathrm{~km} \mathrm{~s}^{-1}$ to all the velocities in the Catalina catalog. We also made use in this study of the latest version of the catalog of halo red giants in the Spaghetti survey (S09 and references herein), as well as M giants from Majewski et al. (2004), most of which are probably associated with the Sgr dSph galaxy. There are also several BHB catalogs available, namely, Sirko et al. (2004); Brown et al. (2008, 2010). All of these catalogs contain stars within or around the region studied in this work, as shown in Fig. 12. We explored also the catalog of BHB stars by De Propris et al. (2010) which overlaps our region. However, these BHB stars are located at distances $>28 \mathrm{kpc}$, and hence they are much farther away than our sample.

In the case of the BHB catalog of Sirko et al. (2004) which is based in SDSS-DR1, we searched for updated radial velocities in SDSS-DR7 in order to overcome any possible systematic error in the radial velocity in this early catalog. In many cases, more than one DR7 spectroscopic measurement was available, and we calculated an error-weighted mean velocity for those stars. On the other hand, the catalog of Brown et al. (2008) is based in 2MASS and, since it is a nearby sample, it may contain thick disk stars as well as halo stars. Following those authors, we eliminated all stars with $z<5 \mathrm{kpc}$ to have a cleaner sample of halo BHBs. We also made sure all radial velocities were in the same frame (the galactic standard of rest) as our sample of RRLS. From Fig. 12, there are no BHB data at declinations south of $\sim-3^{\circ}$, thus the SEKBO, Catalina and Spaghetti samples are specially valuable on those regions.

In order to study which other stars are potentially associated with the groups of RRLS, we determined which RRLS in our significant groups paired with any other star in the additional catalogs, using the same algorithm described above with $\epsilon=0.05$. In this case, however, we did not weight by the errors since the properties of each catalog are very different from each other. The results are shown in Fig. 13. Each row in the figure corresponds to different planes (Dec vs. RA, distance vs. RA, and distance vs. $V_{\mathrm{gsr}}$ ) of the 5 significant groups described in the last section.

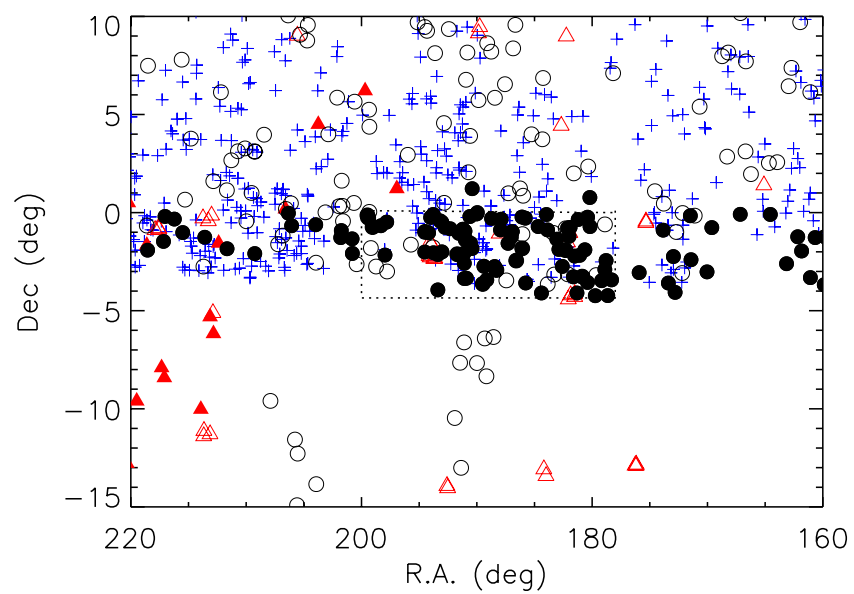

Fig. 12. Distribution in the sky near the Virgo region of halo tracers for which both velocities and distances are available in the literature. Circles represent RRLS (solid circles are stars in the QUEST survey, which includes the ones reported in this work; open circles come from the SEKBO and Catalina surveys), + symbols are BHB stars from Sirko et al. (2004), and Brown et al. (2008, 2010), open and solid triangles are red giants from Starkenburg et al. (2009) and M giants from Majewski et al. (2004), respectively. Notice that the figure shows a region in the sky much larger than the one explored in this work with RRLS, i.e., the dotted rectangle with $178^{\circ}<\mathrm{RA}<200^{\circ},-4^{\circ}<\mathrm{Dec}<0^{\circ}$.

All these groups have RRLS that pair with several BHB stars and red giants (crosses and triangles respectively). Each one of the groups of RRLS have at least one red giant which is associated with it. Similarly, additional RRLS for each group are provided by either the SEKBO or Catalina catalogs. None of the $\mathrm{M}$ giant stars paired with any of our groups. The individual members of each one of these groups are listed in Table 9. The table indicates for each group, the survey from which the member was identified, the type of star used as tracer in that survey (RRLS: RR Lyrae stars, BHB: Blue Horizontal Branch stars, and RG: Red Giant stars), the ID within the survey for the star, its RA and Dec as well as its velocity and heliocentric distance. Finally, the last column gives the corresponding reference. Given the very different properties of the catalogs used, each one having different coverages and completeness, we did not attempt to make simulations to test the significance of those additional members. The additional members suggest possible directions, within the limits of their own surveys, in which follow up observations can focus in the future.

The left columns in Fig. 13 show the spatial distribution of each group (Dec vs. RA). All the groups extend in the sky to some degree in both directions. It is especially noticeable for group D and this is just a consequence of that group being close to the Sun. These plots show us that the five kinematical groups occupy the same region of the sky. The right panels in Fig. 13 show the distribution in phase space for every group. It is clear that each group occupies a very different region in this plane making the case that they are distinct structures.

\subsection{Properties of the groups}

In Table 10 we summarized the main properties of the kinematical groups we have found. The mean properties were calculated using only the RRLS which are members of each group, including the ones from SEKBO, Catalina and from the extended QUEST catalog. 

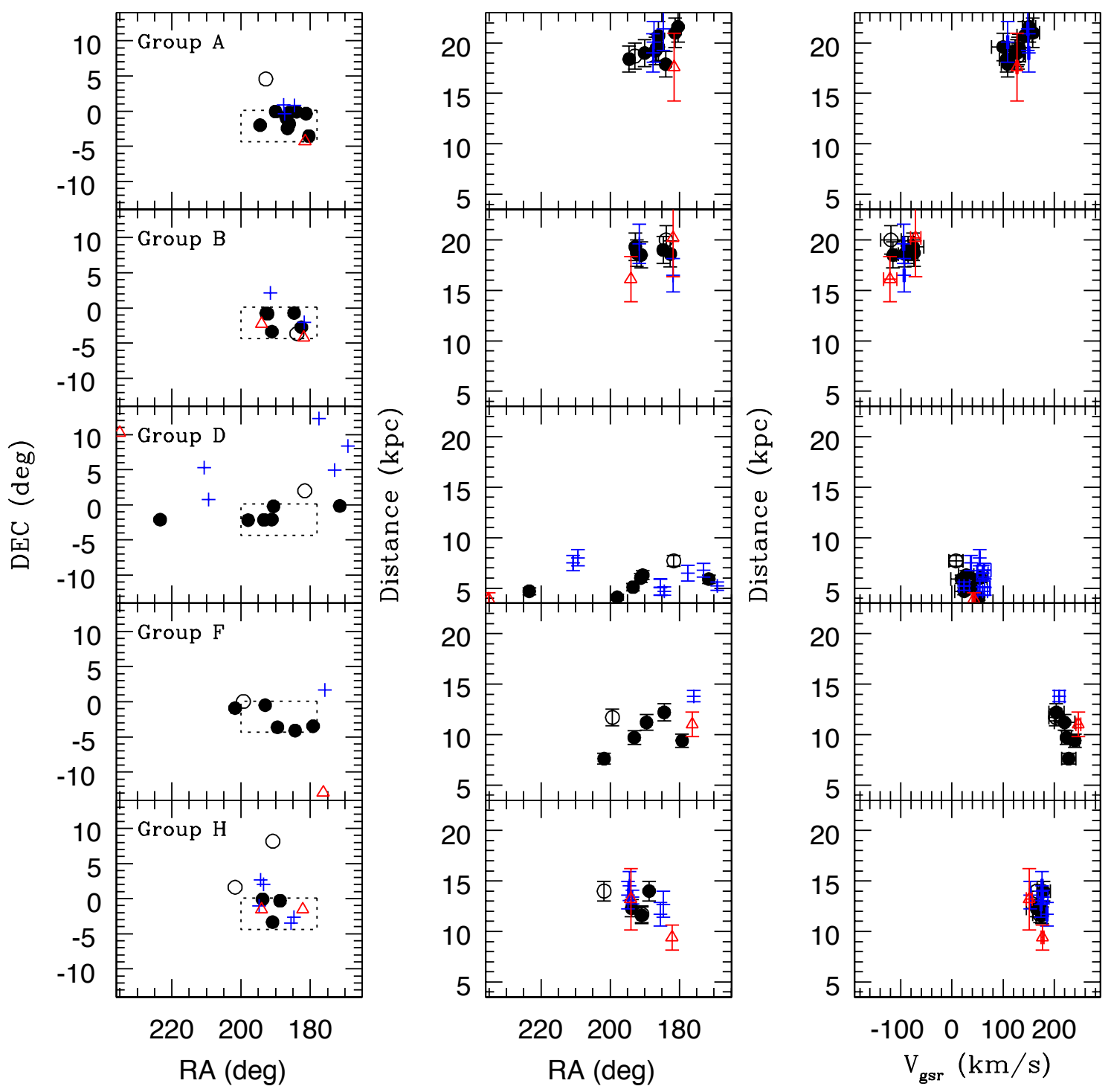

Fig. 13. Distribution in the sky and in phase space of the groups detected in this work. Each row shows different planes for the same group. Symbols are the same as in Fig. 12.

We consider here whether the VSS (group A) may be related with groups $\mathrm{F}$ and $\mathrm{H}$. Figure 13 shows there is a sequence in the location of these 3 groups in phase space (rightmost panels in the figure), with velocity increasing and distance decreasing from group A, to group $\mathrm{H}$, to group $\mathrm{F}$. Table 10 indicates that the properties of the RRLS in these groups are similar and thus, at this point, a relationship among them cannot be discarded. The three groups have similar metallicities, although group A has the largest metallicity dispersion among the three. They also have RRab with similar mean periods, and the type ab dominates over the type $c$ among the RRLS in the three groups.

Carlin et al. (2012) identified turnoff stars with proper motions, in a small region at $(\mathrm{RA}, \mathrm{Dec})=\left(178.8^{\circ},-0.6^{\circ}\right)$, in order to calculate an orbit for the VSS. It is worth noticing that the properties of those turnoff stars (mean distance $=14 \mathrm{kpc}$, $V_{\mathrm{gsr}}=153 \mathrm{~km} \mathrm{~s}^{-1}$ ) are more compatible with stars in group $\mathrm{H}$ rather than the VSS itself. Ideally, obtaining proper motions in more extended and deeper areas will allow to determine whether the two groups have similar orbits. Curiously, QUEST star \#167, which has been associated with the turnoff stars measured by
Carlin et al. (2012) does not match either members of group H or any other star in the rest of the groups. After increasing the value of $\epsilon$ to 0.06 , this star matches 3 members of the VSS (group A) instead.

On the other hand, Newberg et al. (2007) studied a sample of F stars with bluer colors than the ones containing the VSS signature. Among these, one velocity peak of significant relevance is present on the N07 N field at $\gtrsim 150 \mathrm{~km} \mathrm{~s}^{-1}$ and at relatively close distances $(<14.5 \mathrm{kpc})$. Newberg et al. (2007) believed it might be related to the VSS but did not elaborate more on this. We can now connect this feature to our Group H. The presence of this peak on the sample with colors bluer than the VSS turnoff suggest that it is possible that the Group $\mathrm{H}$ is actually composed of a different population and as such, it may be a different feature than the VSS. We notice that this group is not present on the N07 S field, suggesting that it is not as extended as the VSS.

Group B is peculiar among the groups because is dominated by type c stars (only 1 out of the 6 RRLS in the group belongs to the type ab class). On the other hand, group D has the largest ratio of BHB/RRLS, although this may be an observational bias 
Table 9. Individual members of kinematical groups.

\begin{tabular}{llllccccc}
\hline \hline Group & Survey & Tracer & ID & $\begin{array}{c}\text { RA } \\
(\mathrm{deg})\end{array}$ & $\begin{array}{c}\text { Dec } \\
(\mathrm{deg})\end{array}$ & $\begin{array}{c}V_{\mathrm{gsr}} \\
\left(\mathrm{km} \mathrm{s}^{-1}\right)\end{array}$ & $\begin{array}{c}d \\
(\mathrm{kpc})\end{array}$ & Reference \\
\hline A & QUEST & RRLS & 725 & 180.404037 & -3.557914 & 151 & 21 & This work \\
A & QUEST & RRLS & 177 & 181.212601 & -0.351735 & 158 & 21 & This work \\
A & QUEST & RRLS & 189 & 183.965347 & -0.098466 & 109 & 17 & This work \\
A & QUEST & RRLS & 195 & 186.065384 & -1.820641 & 142 & 20 & This work \\
A & QUEST & RRLS & 196 & 186.083054 & -0.227318 & 100 & 19 & This work \\
A & QUEST & RRLS & 740 & 186.586151 & -2.446141 & 131 & 19 & This work \\
A & QUEST & RRLS & 199 & 186.930542 & -0.965226 & 129 & 19 & This work \\
A & QUEST & RRLS & 210 & 190.014893 & -0.069222 & 127 & 19 & This work \\
A & QUEST & RRLS & 237 & 194.513031 & -2.009306 & 107 & 18 & This work \\
A & CSS & RRLS & J125124.4+043347 & 192.851690 & 4.563240 & 129 & 18 & Drake et al. (2013) \\
\hline
\end{tabular}

Notes. This table is available in its entirety at the CDS. A portion is shown here for guidance regarding its form and content. See text for description.

Table 10. Properties of the groups.

\begin{tabular}{lccccccccccc}
\hline \hline Group & $\begin{array}{c}\mathrm{RA} \\
(\mathrm{deg})\end{array}$ & $N_{\mathrm{RR}}$ & $N_{\mathrm{BHB}}$ & $N_{\mathrm{BHB}} / N_{\mathrm{RR}}$ & $R R_{c} / R R_{\mathrm{ab}}$ & $\begin{array}{c}P_{\mathrm{ab}} \\
(\mathrm{d})\end{array}$ & {$[\mathrm{Fe} / \mathrm{H}]$} & $\sigma[\mathrm{Fe} / \mathrm{H}]$ & $\begin{array}{c}\left\langle V_{\mathrm{gsr}}\right\rangle \\
\left(\mathrm{km} \mathrm{s}^{-1}\right)\end{array}$ & $\begin{array}{c}\sigma V_{\mathrm{gsr}} \\
\left(\mathrm{km} \mathrm{s}^{-1}\right)\end{array}$ & $\begin{array}{c}\langle d\rangle \\
\mathrm{kpc}\end{array}$ \\
\hline $\mathrm{A}$ & 186.863 & 10 & 3 & 0.3 & 0.11 & 0.56 & -1.78 & 0.37 & 128 & 18 & 19.6 \\
$\mathrm{~B}$ & 187.879 & 6 & 2 & 0.3 & 5.00 & 0.52 & -1.69 & 0.18 & -94 & 18 & 19.0 \\
$\mathrm{D}$ & 192.792 & 7 & 8 & 1.1 & 0.40 & 0.55 & -1.64 & 0.09 & 32 & 16 & 5.7 \\
$\mathrm{~F}$ & 191.194 & 6 & 2 & 0.3 & 0.00 & 0.59 & -1.62 & 0.19 & 220 & 13 & 10.3 \\
$\mathrm{H}$ & 193.187 & 5 & 1 & 0.2 & 0.00 & 0.57 & -1.72 & 0.21 & 172 & 6 & 12.7 \\
\hline
\end{tabular}

given the availability of a large catalog of nearby BHB stars (Brown et al. 2008). Both groups B and D have the shortest mean periods of the RRab stars among all the groups.

\subsection{Comparison with Sgr models}

Figure 14 (top) shows that the models of Sgr debris by Law \& Majewski (2010) indicate that its tidal streams pass close enough to our area of study that we can expect that some of our RRLS belong to this galaxy. Although most of the Sgr stars in the region are located at distances larger than $\sim 40 \mathrm{kpc}$ (bottom panel in Fig. 14), there are some particles at closer distances, within the range studied in this work. However, the nearby debris does not seem to be related with any of the kinematical groups found in this work, shown as coloured symbols in Fig. 14. None of our groups have either the velocity or the distance for the expected Sgr debris in the region.

There is a small number of nearby Sgr debris stars in this part of the sky (distance $\lesssim 11 \mathrm{kpc},-160 \lesssim V_{\text {gsr }}\left(\mathrm{km} \mathrm{s}^{-1}\right) \lesssim-90$ ). According to Law \& Majewski (2010) this part of the stream is made of stars that were mostly stripped from Sgr between 3.2 to $5.0 \mathrm{Gyr}$ ago. The small number of model particles translates in an even smaller number of RRLS predicted to exist in that nearby stream. Zinn et al. (2014) estimated that for every particle in the model of Law \& Majewski (2010) there are 0.23 RRLS. Thus, with 24 particles in that nearby stream, there should be $\sim 5 \mathrm{Sgr}$ RRLS in the region. While there are 3 RRLS which have similar distance and velocity than the stream (see Fig. 14), we also expect, from the simulations described in Sect. 4.4, $4 \pm 2$ halo RRLS in the same region of the phase space. Thus, there is not a clear excess of RRLS that can be readily associated with this stream from an old wrap. A similar conclusion on the lack of strong evidence for a stream stripped between 3.2 to 5 Gyr ago was made by Zinn et al. (2014) based on the spatial distribution of a larger sample of RR Lyrae stars.
The bottom panel in Fig. 14 also shows the regions where other kinematical signatures have been found by other groups. Brink et al. (2010) and Casey et al. (2012) studied the velocity distribution of main sequence stars and red giants in the region, respectively (see Fig. 3). Although Brink et al. (2010) and Casey et al. (2012) state somewhat different ranges for their velocity features, they are consistent with a mean velocity of $\sim-80 \mathrm{~km} \mathrm{~s}^{-1}$. This velocity is sufficiently close to mean velocity of Group B that they may be related. However, the majority of the stars in both the Brink et al. (2010) and the Casey et al. (2012) samples are probably more distant that $20 \mathrm{kpc}$, and they may instead be related to the Sgr stream, which lies at $\sim 50 \mathrm{kpc}$, or with the substructure recently identified by Jerjen et al. (2013) at $\sim 23 \mathrm{kpc}$, whose velocity has not yet been measured.

\section{Conclusions}

We present the first complete spectroscopic study of all RRLS in the QUEST survey in the direction of the VSS. For this work we have gathered 82 targets combining two previous works by the collaboration (Duffau et al. 2006; Vivas et al. 2008), and reporting on 36 RRLS newly observed stars. The sample covers heliocentric distances between 4-23 kpc, 290 square degrees, and RA between $178^{\circ}-200^{\circ}$. This homogeneous sample enables us to disentangle in phase space several substructures lying along the same line of sight. This is possible because RRLS are excellent standard candles and allow the determination of precise distances.

A summary of all kinematic groups found in the Virgo region is displayed in Table 11. In this table, each check mark $(\sqrt{ })$ under a study reference represents a detection of the corresponding group. The first column contains, in addition to the 5 groups of RRLS detected here, two groups predicted to lie in this region by the models of the disruption of Sgr ("Sgr far" refers to stars located at distances $>40 \mathrm{kpc}$, while "Sgr near" correspond to stars between $\sim 8$ and $18 \mathrm{kpc}$, as shown by the debris (asterisks) in 
Table 11. Kinematic detections in the Virgo region.

\begin{tabular}{lcccccc}
\hline \hline Group & $\begin{array}{c}\text { This work } \\
\text { RRLS }\end{array}$ & $\begin{array}{c}\text { N07 } \\
\text { F-TO }\end{array}$ & $\begin{array}{c}\text { B10 } \\
\text { F-TO }\end{array}$ & $\begin{array}{c}\text { C12 } \\
\text { K-giants }\end{array}$ & $\begin{array}{c}\text { S09 } \\
\text { K-giants }\end{array}$ & $\begin{array}{c}\text { P09 } \\
\text { RRLS }\end{array}$ \\
\hline Group A (VSS) & $\sqrt{ }$ & $\sqrt{ }$ & $\sqrt{ }$ & $\sqrt{ }$ & & $\sqrt{ }$ \\
Group B & $\sqrt{ }$ & $*$ & $\sqrt{ }$ & & & \\
Group D & $\sqrt{ }$ & & $\sqrt{ }$ & $\sqrt{ }$ & & \\
Group F & $\sqrt{ }$ & & $\sqrt{ }$ & & $\sqrt{ }$ & \\
Group H & $\sqrt{ }$ & $\sqrt{ }$ & $\sqrt{ }$ & $\sqrt{ }$ & $\sqrt{ }$ & $\sqrt{ }$ \\
Sgr far & & $\sqrt{ }$ & $*$ & & $\sqrt{ }$ \\
Sgr near & & $\sqrt{ }$ &
\end{tabular}

Notes. Each check mark $\sqrt{ }$ under a study reference represents a detection of the corresponding group.

References. N07 (Newberg et al. 2007); B10 (Brink et al. 2010); C12 (Casey et al. 2012); S09 (Starkenburg et al. 2009); P09 (Prior et al. 2009).
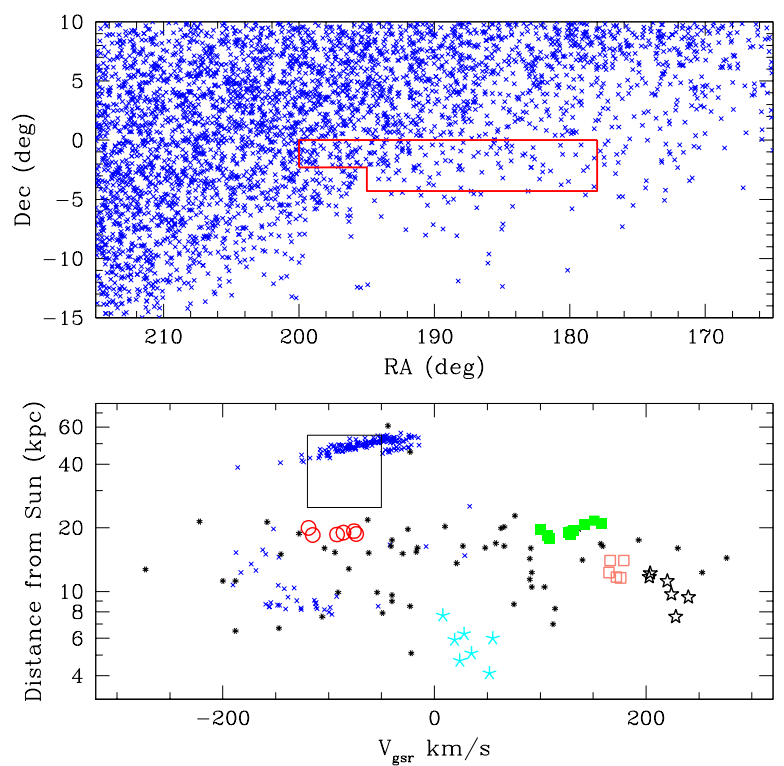

Fig. 14. Top: distribution in the sky around Virgo of the Sgr debris in the models of Law \& Majewski (2010). The polygon indicates the region studied in this work. Bottom: velocity and distance of model particles (crosses) and RRLS (asterisks) within the box indicated above. Stars in each one of the 5 kinematical groups detected here are shown with their corresponding symbol (Table 8). The two RRLS at distances $>40 \mathrm{kpc}$ were taken from Vivas et al. (2005). Rectangle indicates the approximate location of another kinematical group found by Brink et al. (2010) and Casey et al. (2012).

Fig. 14). An asterisk in Table 11 indicates a weak detection of the corresponding group.

The VSS, which is now reconciled with feature S297+63-20.5 (Newberg et al. 2007), is the most significant feature in our sample. It spans at least between 17.9-21.6 kpc along the line of sight and has a mean radial velocity of $128 \mathrm{~km} \mathrm{~s}^{-1}$, as deduced from its RRLS components. A possible relationship of the VSS with two other groups (Groups F and H) cannot be discarded at this point. The three groups form a sequence in phase space with the VSS and group F at the two ends of the sequence with group $\mathrm{H}$ intermediate in both velocity and distance (see Figs. 13 and 14).

Another significant group, composed mostly of type c stars, was found at a similar distance than the VSS, $19 \mathrm{kpc}$, but with a velocity of $-94 \mathrm{~km} \mathrm{~s}^{-1}$. Similar velocity signatures have been found in the region by (Brink et al. 2010) and Newberg et al. (2007), although the latter found it among the brightest stars in their sample, hence, presumably corresponding to a group at a shorter distance than our detection. Finally, a nearby group at only $6 \mathrm{kpc}$ was also detected with a mean velocity of $V_{\mathrm{gsr}}=$ $32 \mathrm{~km} \mathrm{~s}^{-1}$. The properties of this group are harder to isolate due to its much larger angular extension in the sky and its velocity being close to the expected mean velocity of halo stars.

Using the models of Law \& Majewski (2010), we find that while the Sgr stream debris are not expected to be dense in this region at distances closer than $40 \mathrm{kpc}$, some debris is expected to be found in the VSS area. Several of our RRLS lie within the expected distance and velocity of those predictions. However, none of the kinematic groups we found in this work seem to be related with Sgr debris. For the distances involved in this study the dominant feature is the VSS. This was not the case in the works of Brink et al. (2010) and Casey et al. (2012). Their exploration included targets presumably at much larger distances. In those cases, the dominant feature seems to be the Sgr leading stream or another new sub-structure in the region as suggested by Jerjen et al. (2013).

The numerous kinematic features found in our sample of RRLS qualitatively agree with the expectations of halo substructures in cosmological models of galaxy formation. Our results suggest that several streams, rather than a single accretion event, are responsible for the excess of stars found in the Virgo region.

The true shape and extension of all the streams detected in this work will require accurate distances and velocities of targets in a more extended region than the one considered here. The possibility of clarifying which of the different substructures are related to each other and which are not, might require additional information. One possibility is the chemical tagging of their stars, which is one direction we are currently pursuing.

Acknowledgements. We thank the anonymous referee for useful suggestions and comments. We thank Amina Helmi for kindly providing us the data of the red giants found in the Spaghetti survey. We thank Dana CasettiDinescu for kindly providing her orbit for RRL 167. S.D. acknowledges support from both ARI and CIDA during her visit to CIDA, Venezuela in November 2010 to work on this project, and to Yale University de Chile through the joint PhD program (MECESUP UCH0118, Fundacion Andes C13798, Fondap 15010003 and CONICYT 24070078). S.D. also acknowledges support from Sonderforschungsbereich SFB 881 "The Milky Way System" (subprojects A2, A3 and A4) of the German Research Foundation (DFG). R.Z. acknowledges support from National Science Foundation grants AST 05-07364 and AST 1108948. M.T.R and R.M. received partial support from CATA (PB06) CONICYT. A.K.V. is thankful for the hospitality of the Department of Astronomy at University of Michigan during her sabbatical leave in which part of this work was made. This research was based on data obtained at KPNO (Kitt Peak National Observatory, National Optical Astronomy Observatory, which is operated by the Association of Universities for Research in Astronomy (AURA) under cooperative agreement with the National Science Foundation), WIYN (The WIYN Observatory is a joint facility of the University of WIsconsin-Madison, Indiana University, Yale University, and the National Optical Astronomy Observatory), 
CTIO (Cerro Tololo Inter-American Observatory, National Optical Astronomy Observatory, which is operated by the Association of Universities for Reseach in Astronomy, under contract with the National Science Foundation), telescopes at the European Southern Observatory La Silla, Magellan's Clay Telescope at LCO (operated by a consortium consisting of the Carnegie Institution of Washington, Harvard University, MIT, the University of Michigan, and the University of Arizona) and instruments operated by the SMARTS Consortium. We thank the staff at all of these observatories for their help during the time the observations were conducted.

\section{References}

Baltay, C., Snyder, J. A., Andrews, P., et al. 2002, PASP, 114, 780 Bell, E. F., Zucker, D. B., Belokurov, V., et al. 2008, ApJ, 680, 295 Belokurov, V., Zucker, D. B., Evans, N. W., et al. 2006, ApJ, 642, L137

Bonaca, A., Jurić, M., Ivezić, Ž., et al. 2012, AJ, 143, 105 Brink, T. G., Mateo, M., \& Martínez-Delgado, D. 2010, AJ, 140, 1337 Brown, W. R., Beers, T. C., Wilhelm, R., et al. 2008, AJ, 135, 564 Brown, W. R., Geller, M. J., Kenyon, S. J., \& Diaferio, A. 2010, AJ, 139, 59 Bullock, J. S., \& Johnston, K. V. 2005, ApJ, 635, 931

Carlin, J. L., Yam, W., Casetti-Dinescu, D. I., et al. 2012, ApJ, 753, 145 Casetti-Dinescu, D. I., Girard, T. M., Majewski, S. R., et al. 2009, ApJ, 701, L29 Casey, A. R., Keller, S. C., \& Da Costa, G. 2012, AJ, 143, 88

Clewley, L., \& Kinman, T. D. 2006, MNRAS, 371, L11 Cooper, A. P., Cole, S., Frenk, C. S., et al. 2010, MNRAS, 406, 744 De Propris, R., Harrison, C. D., \& Mares, P. J. 2010, ApJ, 719, 1582 Demarque, P., Zinn, R., Lee, Y.-W., \& Yi, S. 2000, AJ, 119, 1398 Drake, A. J., Catelan, M., Djorgovski, S. G., et al. 2013, ApJ, 763, 32 Duffau, S., Zinn, R., Vivas, A. K., et al. 2006, ApJ, 636, L97 Helmi, A., Cooper, A. P., White, S. D. M., et al. 2011, ApJ, 733, L7 Ivezić, Ž., Goldston, J., Finlator, K., et al. 2000, AJ, 120, 963
Jerjen, H., Da Costa, G. S., Willman, B., et al. 2013, ApJ, 769, 14 Jurić, M., Ivezić, Ž., Brooks, A., et al. 2008, ApJ, 673, 864 Keller, S. C. 2010, PASA, 27, 45

Keller, S. C., Murphy, S., Prior, S., Da Costa, G., \& Schmidt, B. 2008, ApJ, 678, 851

Keller, S. C., da Costa, G. S., \& Prior, S. L. 2009, MNRAS, 394, 1045

Law, D. R., \& Majewski, S. R. 2010, ApJ, 714, 229

Layden, A. C. 1994, AJ, 108, 1016

Layden, A. C. 1995, AJ, 110, 2288

Layden, A. C. 1998, AJ, 115, 193

Majewski, S. R., Skrutskie, M. F., Weinberg, M. D., \& Ostheimer, J. C. 2003, ApJ, 599, 1082

Majewski, S. R., Kunkel, W. E., Law, D. R., et al. 2004, AJ, 128, 245

Martínez-Delgado, D., Peñarrubia, J., Jurić, M., Alfaro, E. J., \& Ivezić, Z. 2007, ApJ, 660, 1264

Moultaka, J., Ilovaisky, S. A., Prugniel, P., \& Soubiran, C. 2004, PASP, 116, 693

Newberg, H. J., Yanny, B., Rockosi, C., et al. 2002, ApJ, 569, 245

Newberg, H. J., Yanny, B., Cole, N., et al. 2007, ApJ, 668, 221

Preston, G. W., \& Sneden, C. 2000, AJ, 120, 1014

Prior, S. L., Da Costa, G. S., Keller, S. C., \& Murphy, S. J. 2009, ApJ, 691, 306

Sesar, B., Ivezić, Ž., Lupton, R. H., et al. 2007, AJ, 134, 2236

Sirko, E., Goodman, J., Knapp, G. R., et al. 2004, AJ, 127, 899

Starkenburg, E., Helmi, A., Morrison, H. L., et al. 2009, ApJ, 698, 567

Vivas, A. K. 2002, Ph.D. Thesis, AA, Yale University, USA

Vivas, A. K., \& Zinn, R. 2006, AJ, 132, 714

Vivas, A. K., Zinn, R., Andrews, P., et al. 2001, ApJ, 554, L33

Vivas, A. K., Zinn, R., Abad, C., et al. 2004, AJ, 127, 1158

Vivas, A. K., Zinn, R., \& Gallart, C. 2005, AJ, 129, 189

Vivas, A. K., Jaffé, Y. L., Zinn, R., et al. 2008, AJ, 136, 1645

Walsh, S. M., Willman, B., \& Jerjen, H. 2009, AJ, 137, 450

Wu, C., Qiu, Y. L., Deng, J. S., Hu, J. Y., \& Zhao, Y. H. 2005, AJ, 130, 1640

Xue, X.-X., Rix, H.-W., Yanny, B., et al. 2011, ApJ, 738, 79

Zinn, R., Horowitz, B., Vivas, A. K., et al. 2014, ApJ, 781, 22 
S. Duffau et al.: A comprehensive view of the Virgo stellar stream
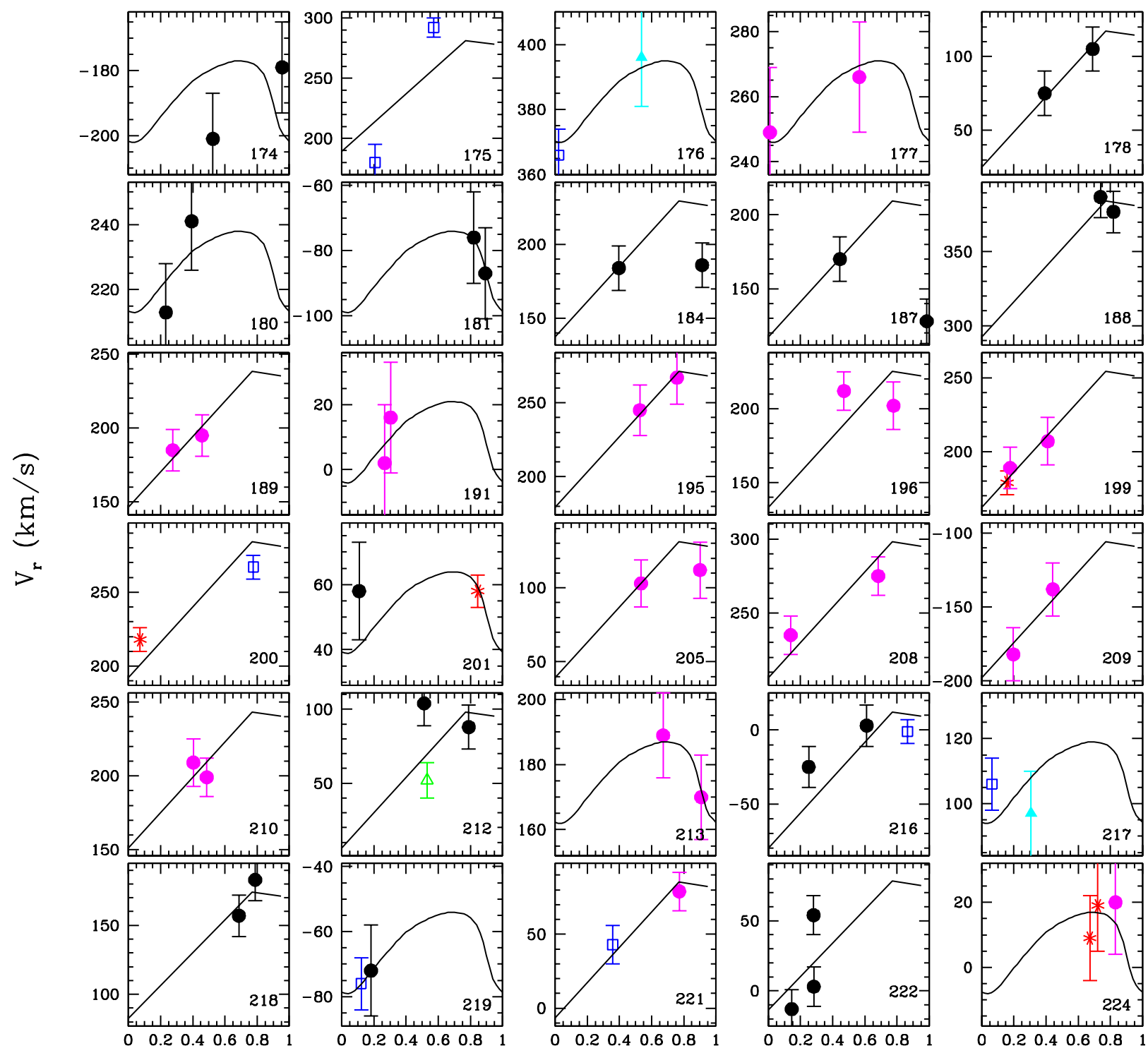

\section{Phase}

Fig. 6. Radial velocity curves for stars having two or more spectroscopic observations. The symbols correspond to different telescopes/setups as follows: Mag05 and Mag07 (solid magenta circles), ESO3.6m (solid cyan triangles), SMARTS (solid black circles), ESO1.5m (open green triangles), WIYN-R (red asterisks), WIYN-B (open blue squares), and WIYN-G (solid yellow squares). The radial velocity curves plotted are indicated by solid lines. All observations are shown, including those not used in the plotting of the radial velocity curve, because they were taken near the phase of maximum light (see text). Although they were not used for the fitting, they lie within the expected range of velocity. 

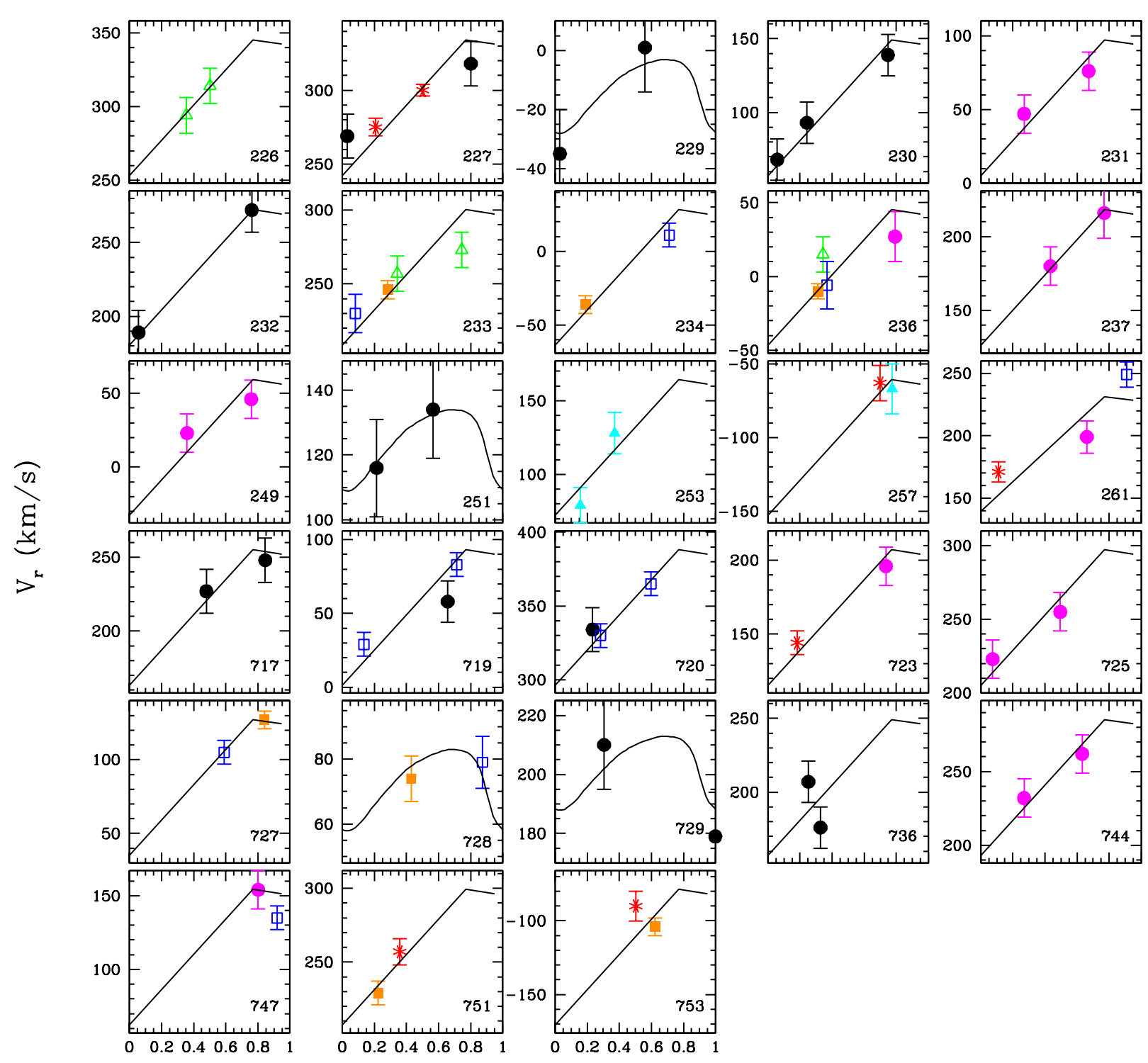

Phase

Fig. 6. continued. 